\title{
Within plate, arc, and collisional Neoproterozoic granitic magmatism in the Araxá Group, Southern Brasília belt, Minas Gerais, Brazil

\author{
Magmatismo granítico Neoproterozoico de ambientes intraplaca, \\ arco e colisão no Grupo Araxá, Faixa Brasília Meridional, \\ Minas Gerais, Brasil
}

Hildor José Seer ${ }^{1 *}$, Lúcia Castanheira de Moraes ${ }^{1}$

\begin{abstract}
Three Neoproterozoic granitic episodes associated with the Araxá Group, in Southern Brasília Belt between the cities of Araxá and Cascalho Rico, Minas Gerais State, Brazil, are characterized from the geologic, geochemical, and geochronological points of view. The earliest episode, of $833 \mathrm{Ma}$, generated in a within-plate environment, is represented by the Quebra Anzol Granite, which has: $\operatorname{low}^{87} \mathrm{Sr} /{ }^{86} \mathrm{Sr}=$ 0.706 ratios and $\varepsilon_{\mathrm{Nd}(833)}=-3.98$; high levels of $\mathrm{SiO}_{2}, \mathrm{Na}_{2} \mathrm{O}, \mathrm{MnO}$, $\mathrm{Nb}, \mathrm{Y}$ and $\mathrm{Co}$; and low concentrations of $\mathrm{Sr}$ and $\mathrm{Ba}$, with the absence of anorthite and presence of diopside, acmite, and positive $\mathrm{Eu}$ anomaly, therefore it may have been originated from mantle sources with small crustal contamination. The second episode, of $790 \mathrm{Ma}$, is represented by the pre-collisional Monte Carmelo Granitic Complex, which was partially remobilized in a collisional event around $630 \mathrm{Ma}$. It is metaluminous to peraluminous with $\operatorname{low}^{87} \mathrm{Sr} /{ }^{86} \mathrm{Sr}$ ratios of 0.706 , high light rare earth elements fractionation relative to heavy rare earth elements, and negative $\mathrm{Nb}, \mathrm{Ta}, \mathrm{Sr}, \mathrm{P}$, and Ti anomalies. Their $\varepsilon_{\mathrm{Nd}(790)}=$ -2.218 , with $\mathrm{T}_{\mathrm{DM}}=1.29 \mathrm{Ga}$, indicate an origin from juvenile sources and reveal the existence of a late Mesoproterozoic magmatic arc in this region. The last granitic episode has taken place in a collisional tectonic environment between 642 and $630 \mathrm{Ma}$, generating peraluminous granites with muscovite, garnet, and tourmaline denominated Serra Velha, Tamanduá, Pirapetinga, Galheirinho, Perdizes, Estrela do Sul, and Cascalho Rico. They have negative $\varepsilon_{\mathrm{Nd}}$, Nd model ages from Neo to Mesoproterozoic, and variable rare Earth elements enrichment patterns, with strong negative Eu anomalies.
\end{abstract}

KEYWORDS: Southern Brasília Belt; Araxá Group; Neoproterozoic; within plate granites; arc granites; collisional granites.
RESUMO: Três episódios de granitogênese Neoproterozoica associados com o Grupo Araxá, na Faixa Brasilia Meridional, são caracterizados geológica, geoquímica e geocronologicamente entre Araxá e Cascalho Rico, em Minas Gerais, no Brasil. O episódio mais antigo, de 833 Ma, gerado em ambiente intraplaca, é representado pelo Granito peralcalino Quebra Anzol, que possui: baixas razóes ${ }^{87} \mathrm{Sr}^{86} \mathrm{Sr}$ de 0,706 e $\varepsilon_{N d(833)}=-3,98$, com elevados valores de $\mathrm{SiO}_{2}, \mathrm{Na}_{2} \mathrm{O}, \mathrm{MnO}, \mathrm{Nb}, \mathrm{Y}$ e Co e baixas concentraçóes de Sr e Ba, tendo ausência de anortita e presença de acmita e diopsídio e anomalia Eu positiva, o que indica sua origem a partir de fontes mantélicas com pequena contaminação crustal. O segundo episódio, de $790 \mathrm{Ma}$, é representado pelo Complexo Granitico Monte Carmelo, que é pré-colisional e parcialmente remobilizado em evento colisional de $630 \mathrm{Ma}$. É metaluminoso a peraluminoso, com baixas razōes ${ }^{87} \mathrm{Sr}{ }^{86} \mathrm{Sr}$ de 0,706 , elevado fracionamento de terras raras leves e anomalias negativas de $\mathrm{Nb}, \mathrm{Ta}, \mathrm{Sr}, \mathrm{P} e$ Ti. Seu $\varepsilon_{N d(790)}$ de $-2,218$ e sua $T_{D M}$ de $1,29 \mathrm{Ga}$ indicam origem a partir de fontes juvenis do final do Mesoproterozoico, de modo similar à dos granitos do Arco Magmático de Goiás, sendo representante de um arco magmático nesta regiāo. O último episódio ocorreu em contexto colisional, entre 642 e $630 \mathrm{Ma}$, gerando os granitos peraluminosos com muscovita, granada e turmalina, chamados de Serra Velha, Tamanduá, Pirapetinga, Galheirinho, Perdizes, Estrela do Sul e Cascalho Rico. Possuem $\varepsilon_{N d t}$ negativos, idades modelos de Nd Neo a Mesoproterozoicas e padróes de enriquecimento de terras raras variáveis, com fortes anomalias negativas de Eu.

PALAVRAS-CHAVE: Faixa Brasilia Meridional; Grupo Araxá; Neoproterozoico; granitos intraplaca; granitos de arco; granitos colisionais.

${ }^{1}$ Centro Federal de Educação Tecnológica de Minas Gerais - CEFET-MG, Campus Araxá, Araxá (MG), Brasil. E-mail: hildorster@gmail.com, luciacm_mg@yahoo.com.br *Corresponding author

Manuscrito ID 28703. Recebido em: 28/08/2012. Aprovado em: 12//04/2013 


\section{INTRODUCTION}

Granitic rocks that are intrusive into quartz-mica schist and amphibolites of the Araxá Group, Southern Brasília Belt (SBB), usually occur between the cities of Araxá and Cascalho Rico, Minas Gerais State, in Brazil (Fig. 1). The earliest reference to these granites is by Barbosa (1937). After some time, Barbosa et al. (1970) drew the attention to the existence of granites and gneisses from the region of Araxá up to Estrela do Sul and Abadia dos Dourados. Hasui and Almeida (1970) performed the first K-Ar dating of granites and metamorphic rocks of this area, while Besang et al. (1977) studied the Quebra Anzol and Monte Carmelo granites by Rb-Sr geochronology. Brod et al. (1991) described intrusive granites into mica schists and metabasic rocks of Araxá Group near Abadia dos Dourados. The first results related to granites associated with the Araxá Group in its type region were performed by Seer (1999), Seer and Dardenne (2000) and Seer et al. (2001). Valeriano et al. (2004b) presented the first U-Pb dating of an Araxá granite obtaining the age of $637 \mathrm{Ma}$, which confirm its relation with the Brasiliano Cycle in the region. Seer et al. (2005) detailed the geology, petrography, and geochemistry of intrusive granites into the Araxá Group in Araxá linking them to collisional magmatism. In 2008, Seer et al. reported the first U-Pb dating of Estrela do Sul Granite, whereas in 2010 the preliminary results of geochronological analyses through the U-Pb and $\mathrm{Sm}-\mathrm{Nd}$ methods of eight granite samples situated between Monte Carmelo and Araxá were presented. In Cataláo, Klein (2008), to the North-western area discussed in this work, studied orthogneiss and granite combining $\mathrm{U}-\mathrm{Pb}$ geochronology and $\mathrm{Sm}-$ $\mathrm{Nd}$ to the whole-rock chemistry.

These works bring to light an evolutionary scenario for the Neoproterozoic SBB showing two main magmatic peaks, one at ca. $790 \mathrm{Ma}$ and the other at ca. $630 \mathrm{Ma}$, reinforcing the evidence raised by other authors in the state of Goiás (Pimentel e Fuck 1992, Pimentel et al. 1992, 1999a, Laux et al. 2005). However, the discovery of Nova Aurora orthogneiss terrain with 1.2 Ga (Klein 2008), in Southeastern Goiás, tectonically placed beside more younger terrains, and within plate granites of $833 \mathrm{Ma}$ in the region of Araxá, reinforces the suggestion that the SBB resulted from the amalgamation of tectonostratigraphic terrains with distinct ages and origins, as suggested by Seer (1999). This work presented and discussed the results of chemical analyses, U-Pb dating and $\mathrm{Sm}-\mathrm{Nd}$ analyses of granites

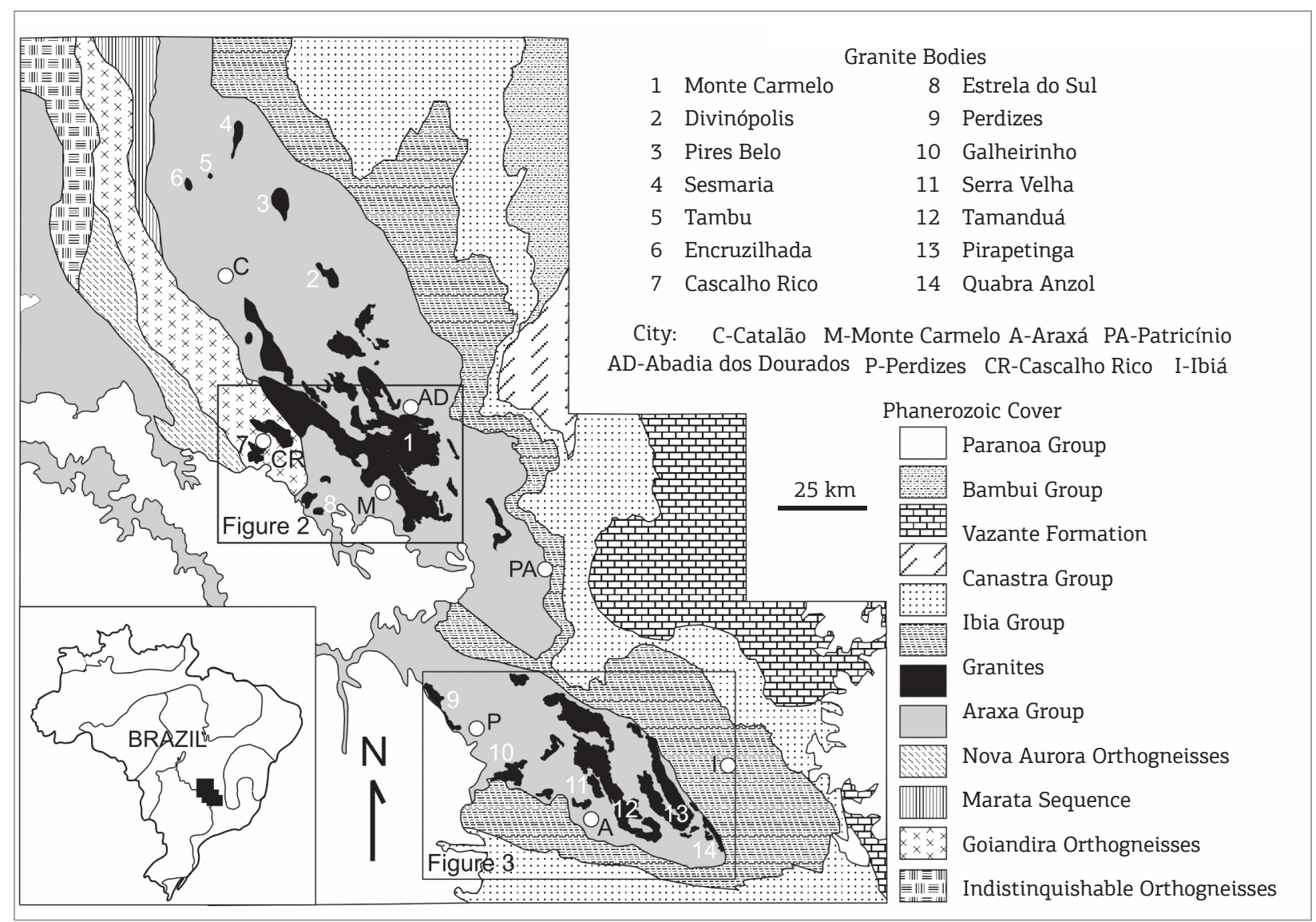

Figure 1. Geological map of Southeast portion of the Brasília Belt showing the granitoids in the Araxá Group. 
from Araxá Group starting in Araxá to Monte Carmelo and Cascalho Rico regions, on the border of Minas Gerais and Goiás States (Figs. 2 and 3).

\section{REGIONAL GEOLOGY}

Between Abadia dos Dourados and Cascalho Rico, the rocks of Araxá Group compose three thrust slices separated by NW-striking shear zones (Seer et al. 2007), as see in Fig. 2. The Western thrust, characterized as upper and that is in tectonic contact with Nova Aurora orthogneisses to the West (Klein 2008), is represented by inter-bedded mafic igneous, ultramafic and clastic sedimentary rocks metamorphosed in amphibolite facies conditions. The lithological types include mica schists including varying proportions of garnet, muscovite, and biotite, with variable amounts of quartz, fine quartzite,

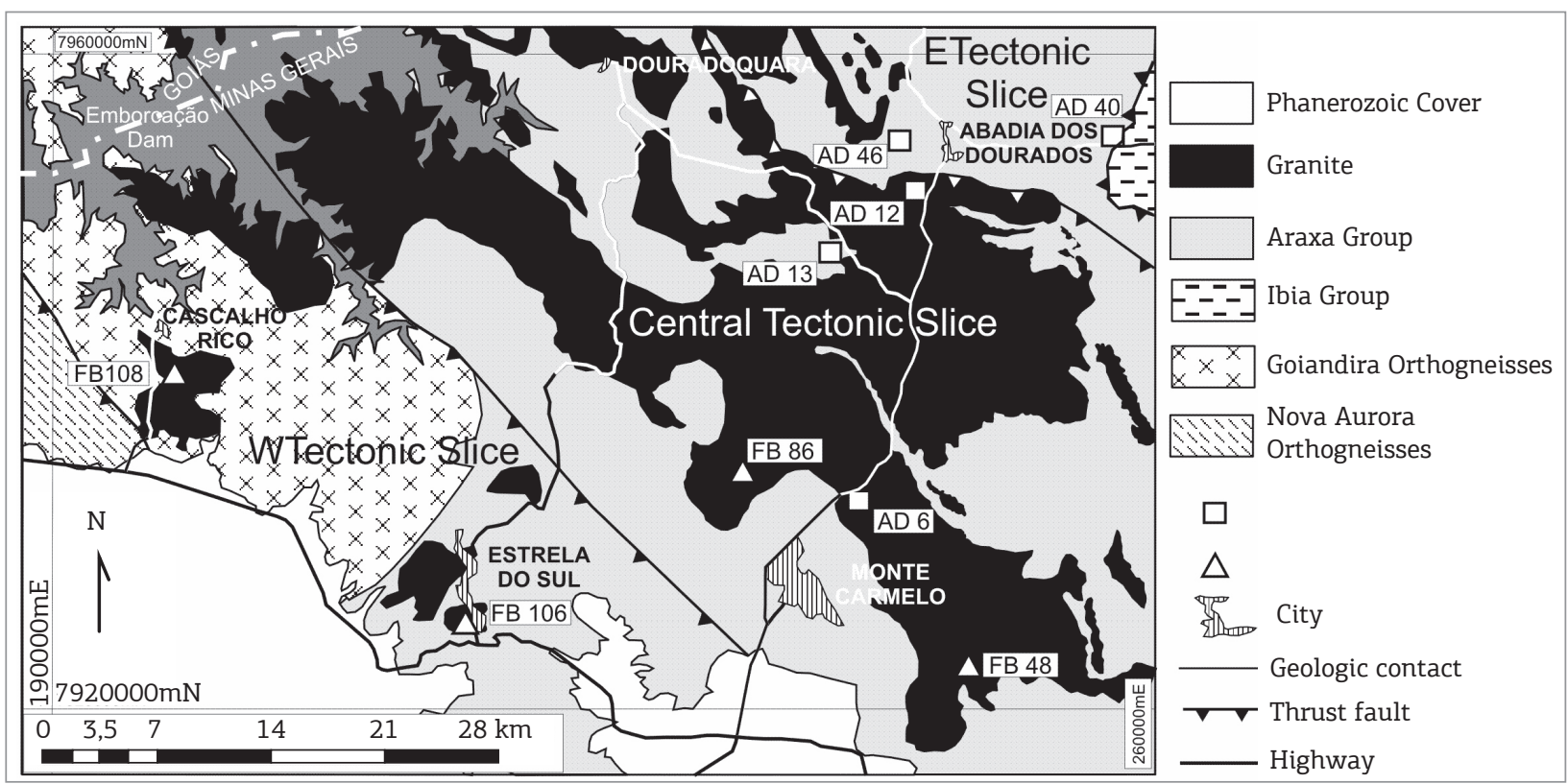

Figure 2. Geological map of Monte Carmelo and Abadia dos Dourados regions with localization of studied samples.

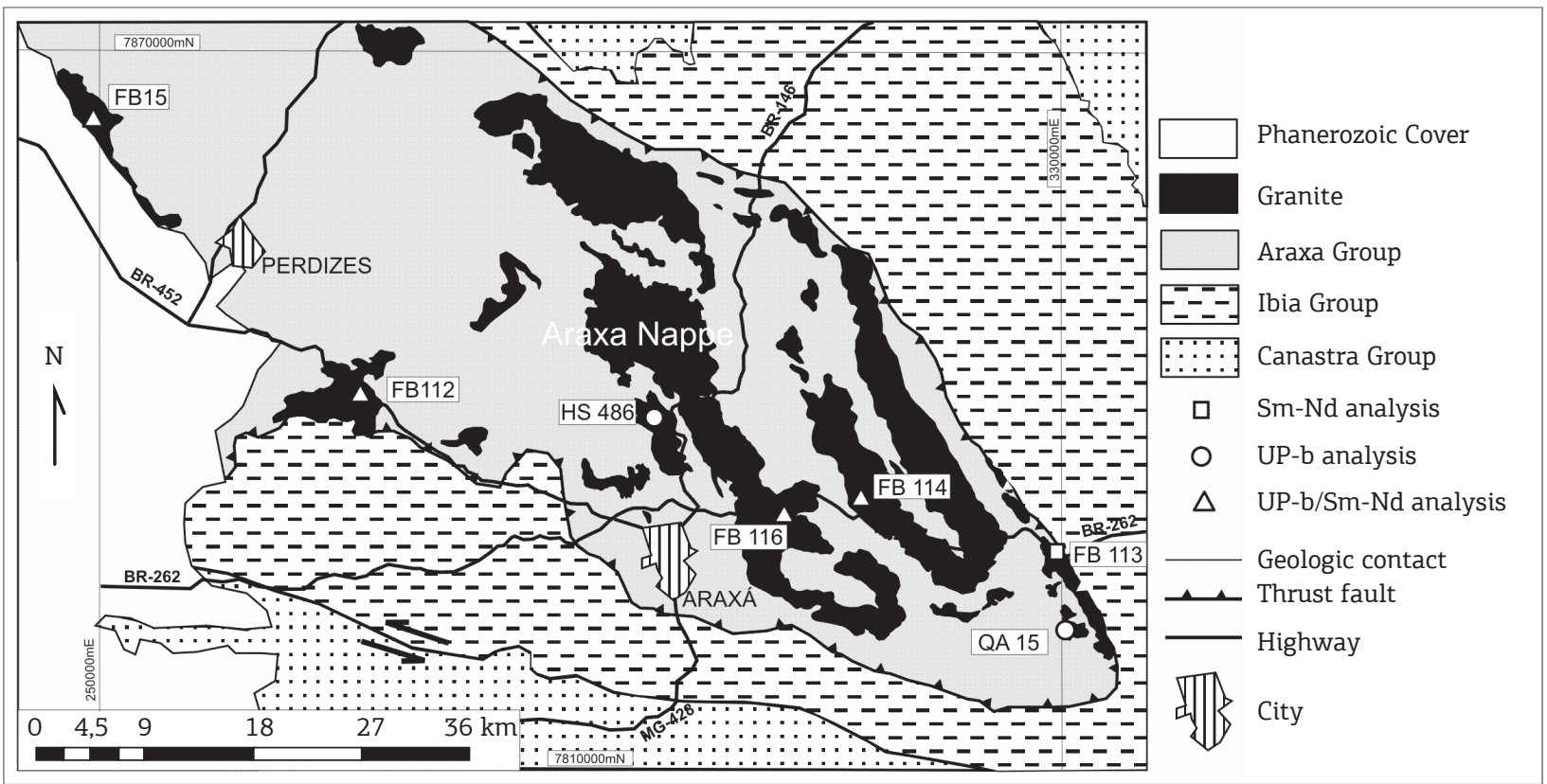

Figure 3. Geological map of the Araxá Nappe region with localization of studied samples. 
coarse- and medium-grained amphibolite, garnet amphibolite, serpentinite, talc-chlorite and tremolite-actinolite schists. They are intruded by several pegmatites and granitic rocks, such as the Estrela do Sul and Cascalho Rico granites (Fig. 2). Medium- and coarse-grained amphibolites and amphibole-chlorite schist intruded by pegmatites and granites are predominant in the intermediate thrust. Monte Carmelo Granitic Complex (Fig. 2), with batholitic proportions and that is deformed, is part of this thrust. The Eastern one is the lower thrust and makes contact with chlorite schists of the Ibiá Group to the East.

The Eastern thrust is a volcanosedimentary sequence of Abadia dos Dourados (Brod et al. 1991), characterized by intercalations of medium to fine amphibolites (metabasalt chemically similar to oceanic ridge basalts), quartz-mica schist (acid metavolcanic with bi-pyramidal quartz), garnet-quartz-mica schist, carbonaceous schist, quartzite and ferruginous meta-chert, intruded by granites and pegmatites of smaller size and number compared to others. The three tectonic slices represent ophiolitic and magmatic arc terrains dismembered and intruded by granitoids and, although deformed, their rocks are relatively well preserved compared to the Araxá Group in the Araxá Nappe (Simóes e Navarro 1996), as seen in Fig. 3.

The granitoids show intense deformation, but are well preserved in the inner portions. In most outcrops a major shallow-dipping foliation was identified, commonly associated with mineral and/or stretching lineation and small isoclinal folds. Kinematic indicators show top-to-the-ESE tectonic transport. A subvertical foliation, related to the development of NW-striking, sinistral transcurrent faults associated with open folds, interfere on the previous structures. The metamorphic and deformational styles in the region of Monte Carmelo are similar to those of the Araxá Nappe (Seer 1999) and to the tectonic compartments of Southeast Goiás (Simôes 2005, Klein 2008). In the Araxá Nappe, the sub-horizontal shear deformation and retrometamorphism, generated during tectonic transport, were more intense, being the individualization of tectonic thrust slices more difficult to identify. Yet, there is a regional zoning, in which ultramafic rocks occur more to the West, and the volcanosedimentary ones farther to the East like in the Monte Carmelo region. Sincollisional granites are predominant in relation to within-plate ones.

\section{ANALYTICAL PROCEDURES}

Nine samples of granitic bodies called Quebra Anzol, Pirapetinga, Tamanduá, Galheirinho, Perdizes, Estrela do Sul, Cascalho Rico, following Seer's (1999) nomenclature
(Seer et al. 2007, 2008), and Monte Carmelo Granitic Complex (Besang et al. 1977, Pereira et al. 1983), were examined using the U-Pb and $\mathrm{Sm}-\mathrm{Nd}$ methods (Tab. 1). The zircon concentrates were extracted by means of gravimetric and magnetic conventional separation techniques and selected by hand picking with binocular microscope. The U-Pb analyzes were performed using the laser ablation inductively coupled plasma mass spectrometry (LA-MC-ICPMS) and the Neptune spectrometer (thermo Finnigan Neptune multi-collector ICPMS) of the Geochronology Laboratory of the University of Brasília, following the method described by Bühn et al. (2009). Nine samples of granite were selected for $\mathrm{Sm}-\mathrm{Nd}$ analyzes in whole-rock, carried out in the same place mentioned, using a mass spectrometer Finnigan MAT262 Multicollector, following the method described by Gioia and Pimentel (2000) and detailed by Della Giustina et al. (2011). Model ages were calculated applying the De Paolo's (1981) model. Some samples were also analyzed for major and minor elements in the Acme Analytical Laboratories Ltd. (Canada) in order to complement the data obtained by Seer (1999).

\section{RESULTS}

The results of the chemical analyzes are presented in Tab. 2. Sm-Nd outcomes are in Tab. 3 and the U-Pb ones are in Tabs. 4 to 10. For the Quebra Anzol Granite, the FB113 sample was investigated for its $\mathrm{Sm}-\mathrm{Nd}$ since it did not possess zircon, while the samples QA-15-1 and QA-15-2 were analyzed only by $\mathrm{U}-\mathrm{Pb}$ method. Unpublished $\mathrm{Sm}-\mathrm{Nd}$ analyzes (initiative of professor doctor Reinhard A. Fuck) of a garnet mica schist and a fine amphibolite (metabasalt) from the volcanosedimentary sequence Abadia dos Dourados (AD-40 and AD-46), a coarse metagabbro of the Chapada das Perdizes Complex (AD-13) hosting the Monte Carmelo Granitic Complex, were added in this paper. Each granitic body was separately described.

\section{Quebra Anzol Granite}

Quebra Anzol Granite is an elongated and deformed body that occurs in the frontal part of the Araxá Nappe and is associated with significant volume of fine to coarse amphibolites (gabbros to basalts) and meta-chert (Seer 1999), as seen in Figs. 1 and 3. It is equigranular to porphyritic, from light to pinkish grey, with orthoclase phenocrysts up to $2 \mathrm{~cm}$ in matrix of quartz, orthoclase and microcline, plagioclase, and biotite. Hornblende, zircon, titanite, and opaque occur as accessories. Albite, sericite, fluorite, chlorite, epidote, carbonate, tourmaline and leucoxen as secondaries of hydrothermal and/or metamorphic 
Table 1. Studied samples with indication of coordinates, modal classification, and analytical procedures

\begin{tabular}{l|c|c|c|c|c|c|c}
\hline Sample & E coord & N coord & Name/Unit & Modal classification & Chem An & U-Pb & Sm-Nd \\
\hline FB-15 & 250258 & 7867987 & Perdizes & sienogranite & & $\mathrm{x}$ & $\mathrm{x}$ \\
\hline FB-48 & 245832 & 7922351 & Monte Carmelo & sienogranite & $\mathrm{x}$ & $\mathrm{x}$ & $\mathrm{x}$ \\
\hline FB-60 & 247406 & 7925122 & Monte Carmelo & monzogranite & $\mathrm{x}$ & & \\
\hline FB-86 & 231824 & 7934524 & Monte Carmelo & tonalite & $\mathrm{x}$ & $\mathrm{x}$ & $\mathrm{x}$ \\
\hline FB-98 & 213621 & 7951587 & Monte Carmelo & granodiorite & $\mathrm{x}$ & & \\
\hline FB-106 & 216088 & 7925046 & Estrêla do Sul & sienogranite & $\mathrm{x}$ & $\mathrm{x}$ & $\mathrm{x}$ \\
\hline FB-108 & 195824 & 7941548 & Cascalho Rico & monzogranite & $\mathrm{x}$ & $\mathrm{x}$ & $\mathrm{x}$ \\
\hline FB-112 & 273162 & 7845530 & Galheirinho & alkali-granite & $\mathrm{x}$ & $\mathrm{x}$ & $\mathrm{x}$ \\
\hline FB-113 & 330434 & 7832718 & Quebra Anzol & granodiorite & & & $\mathrm{x}$ \\
\hline FB-114 & 313040 & 7837435 & Pirapetinga & alkali-granite & $\mathrm{x}$ & $\mathrm{x}$ & $\mathrm{x}$ \\
\hline FB-116 & 308140 & 7837435 & Tamanduá & sienogranite & & $\mathrm{x}$ & $\mathrm{x}$ \\
\hline QA-15 & 329643 & 7830019 & Quebra Anzol & granodiorite & & $\mathrm{x}$ & \\
\hline AD-6 & 239394 & 7932910 & Monte Carmelo & granite & & & $\mathrm{x}$ \\
\hline AD-12 & 245334 & 7952530 & Monte Carmelo & granite & & & $\mathrm{x}$ \\
\hline AD-13 & 240234 & 7947520 & Chapada Perdizes & amphibolite & & & $\mathrm{x}$ \\
\hline AD-40 & 254559 & 7954837 & VSAD & garnet-mica schist & & & $\mathrm{x}$ \\
\hline AD-46 & 243538 & 7955389 & VSAD & amphibolite & & & $\mathrm{x}$ \\
\hline HS-56a & 330800 & 7805600 & Quebra Anzol & monzogranite & $\mathrm{x}$ & & \\
\hline HS-205a & 331200 & 7805600 & Quebra Anzol & alkali-granite & $\mathrm{x}$ & & \\
\hline HS-285b & 330100 & 7805700 & Quebra Anzol & granite & $\mathrm{x}$ & & \\
\hline HS-425a & 329200 & 7806500 & Quebra Anzol & alkali-granite & $\mathrm{x}$ & & $\mathrm{x}$ \\
\hline HS-460a & 299400 & 7822500 & Serra Velha & monzogranite & $\mathrm{x}$ & & \\
\hline HS-486b & 300200 & 7818000 & Serra Velha & monzogranite & $\mathrm{x}$ & $\mathrm{x}$ & \\
\hline
\end{tabular}

FB samples were analyzed at Acme Laboratories Ltd. and HS ones at the Geochemistry Laboratory at Universidade de Brasília (Seer 1999). U-Pb and Sm$\mathrm{Nd}$ analyses were done in the Geocronology Laboratory at Universidade de Brasília, VSAD: volcano-sedimentary sequence Abadia dos Dourados

origins. Garnet happens as broken xenocrysts, with atoll texture and biotite cores. It contains petrographic facies ranging from granodiorite to monzogranite and alkaligranite (Fig. 4), generally displayed from protomylonitic to mylonitic textures. It features rare mafic microgranular enclaves and has levels of $\mathrm{SiO}_{2}$ from 73.2 to $74.69 \%$ and $\mathrm{Al}_{2} \mathrm{O}_{3}$ from 11.89 to $12.47 \%$, quite consistent, high $\mathrm{Na}_{2} \mathrm{O}, \mathrm{K}_{2} \mathrm{O}$ and $\mathrm{CaO}$, including acmite, diopside and normative titanite and absence of anorthite (Tab. 2).

The low values of $\mathrm{TiO}_{2}$, and high ones of $\mathrm{MnO}$, and variable $\mathrm{Na}_{2} \mathrm{O} / \mathrm{K}_{2} \mathrm{O}$ ratios from 0.58 to 2.03 , high $\mathrm{Nb}, \mathrm{Y}$ and $\mathrm{Co}$ and low $\mathrm{Sr}, \mathrm{Ba}, \mathrm{Cr}$ and V (Figs. 5 and 6) stand out. In the AnxAbxOr diagram, the HS-285b sample plots in the field of trondjemites on the boundary of granodiorites, possibly due to the high $\mathrm{Na}_{2} \mathrm{O}$ content and abundant albite, and others in the field of granite (Fig. 7). Two samples have peralcaline character, one is in a transition to metaluminous and the other is metaluminous (Fig. 8). The light rare earth elements (LREE) have low fractionation in relation to the heavy rare earth elements - HREE $\left(\mathrm{La}_{\mathrm{N}} / \mathrm{Yb}_{\mathrm{N}}=21.39\right.$ to 21.74$)$ and the $\mathrm{Eu} / \mathrm{Eu}^{*}$ ratio $=1.71$, representing slight positive $\mathrm{Eu}$ anomaly (Fig. 9). In the multi-element diagram, although some elements have not been analyzed, the higher values of $\mathrm{Nb}$ indicate the absence of subduction zone component (Fig. 10). It is plotted in the field of anorogenic granites in the R1xR2 diagram (Fig. 11) and in that of within plate granites in the NbxY diagram (Fig. 12). Based on Barbarin (1999), it may be classified as PAG type (peralkaline and alkaline granite). Four zircon grains from the QA-15-1 sample provided a concordia age of $834 \pm 6.1 \mathrm{Ma}$ and, in the QA-15-2 sample, four ones provided $831.9 \pm 4.5 \mathrm{Ma}$ (Fig. 13, which was interpreted as an average age of $833 \mathrm{Ma}$ for the body crystallization. Its low ${ }^{87} \mathrm{Sr} /{ }^{86} \mathrm{Sr}$ ratio of 0.706 (Besang et al. $1977), \varepsilon_{\mathrm{Nd}(833)}$ of -3.98 , and geochemical data indicate that it may have been originated from mantle sources with little crustal contamination, although it was affected by post-magmatic hydrothermal alteration and mylonitization.

\section{Monte Carmelo Granitic Complex}

Monte Carmelo Granitic Complex comprises a batholith between the cities of Monte Carmelo and Abadia dos Dourados (Figs. 1 and 2). It is associated with large volume of medium to coarse amphibolites (Chapada das Perdizes Complex, Brod et al. 1991) and is intensely deformed mainly on its borders. Four significant samples of this batholith were investigated and two were analyzed only for Sm-Nd. In the FB-86 and FB-98, the granites were 
Table 2. Chemistry analysis results and calculated normative minerals

\begin{tabular}{|c|c|c|c|c|c|c|c|c|c|c|c|c|c|c|}
\hline Sample & $\begin{array}{c}\text { FB - } \\
48\end{array}$ & $\begin{array}{c}\text { FB - } \\
60\end{array}$ & $\begin{array}{c}\text { FB - } \\
86\end{array}$ & $\begin{array}{c}\text { FB - } \\
98\end{array}$ & $\begin{array}{l}\text { FB - } \\
106\end{array}$ & $\begin{array}{c}\text { FB - } \\
108\end{array}$ & $\begin{array}{l}\text { FB - } \\
112\end{array}$ & $\begin{array}{l}\text { FB - } \\
114\end{array}$ & $\begin{array}{c}\text { HS- } \\
460 a\end{array}$ & $\begin{array}{c}\text { HS- } \\
486 \mathrm{~b}\end{array}$ & $\begin{array}{l}\text { HS- } \\
56 \mathrm{~b}\end{array}$ & $\begin{array}{c}\text { HS- } \\
205 a\end{array}$ & $\begin{array}{c}\text { HS- } \\
285 b\end{array}$ & $\begin{array}{c}\text { HS- } \\
425 a\end{array}$ \\
\hline $\mathrm{SiO}_{2}$ & 70.94 & 69.39 & 70.41 & 66.05 & 72.37 & 66.45 & 75.68 & 70.66 & 73.98 & 75.74 & 74.69 & 74.60 & 73.97 & 73.20 \\
\hline $\mathrm{TiO}_{2}$ & 0.24 & 0.62 & 0.36 & 1.13 & 0.27 & 0.87 & 0.07 & 0.31 & 0.16 & 0.32 & 0.05 & 0.06 & 0.3 & 0.24 \\
\hline $\mathrm{Al}_{2} \mathrm{O}_{3}$ & 15.03 & 14.81 & 14.59 & 15.45 & 14.15 & 15.21 & 13.14 & 14.6 & 11.83 & 12.19 & 12.4 & 12.47 & 11.89 & 12.08 \\
\hline $\mathrm{Fe}_{2} \mathrm{O}_{3}$ & 2.04 & 3.32 & 2.58 & 3.97 & 1.66 & 4.27 & 1.65 & 2.07 & 2.22 & 3.17 & 0.96 & 1.10 & 2.23 & 2.18 \\
\hline $\mathrm{MnO}$ & 0.06 & 0.05 & 0.06 & 0.03 & 0.04 & 0.05 & 0.02 & 0.04 & 0.05 & 0.05 & 0.18 & 0.18 & 0.02 & 0.12 \\
\hline $\mathrm{MgO}$ & 0.49 & 0.82 & 0.81 & 0.99 & 0.34 & 0.97 & 0.16 & 0.5 & 0.57 & 0.72 & 0.06 & 0.10 & 0.79 & 0.42 \\
\hline $\mathrm{CaO}$ & 1.78 & 1.72 & 2.85 & 2.89 & 1.19 & 2.36 & 0.1 & 0.39 & 0.63 & 0.89 & 0.66 & 0.33 & 2.81 & 0.63 \\
\hline $\mathrm{Na}_{2} \mathrm{O}$ & 3.64 & 2.54 & 4.13 & 3.11 & 2.75 & 3.04 & 1.97 & 1.25 & 2.72 & 2.43 & 4.81 & 5.19 & 3.76 & 3.41 \\
\hline $\mathrm{K}_{2} \mathrm{O}$ & 4.87 & 5.12 & 2.54 & 3.89 & 6.05 & 4.58 & 5.49 & 8.35 & 5.03 & 4.58 & 4.26 & 5.21 & 1.85 & 5.79 \\
\hline $\mathrm{P}_{2} \mathrm{O}_{3}$ & 0.14 & 0.24 & 0.1 & 0.4 & 0.14 & 0.34 & 0.05 & 0.32 & 0.26 & 0.1 & 0.03 & 0.03 & 0.06 & 0.12 \\
\hline LOI & 0.6 & 1.1 & 1.4 & 1.7 & 0.9 & 1.3 & 1.7 & 1.3 & 1.72 & 1.82 & 1.0 & 0.7 & 1.64 & 1.1 \\
\hline TOTAL & 99.82 & 99.74 & 99.83 & 99.64 & 99.82 & 99.42 & 100 & 99.75 & 99.01 & 99.13 & 99.01 & 99.90 & 99.17 & 99.10 \\
\hline $\mathrm{Rb}$ & 197.3 & 289.4 & 88.4 & 180.6 & 327.2 & 204.7 & 562.6 & 338.9 & - & - & - & - & - & - \\
\hline $\mathrm{Ba}$ & 871 & 1216 & 871 & 1932 & 1032 & 2585 & 69 & 1623 & 59 & 116 & 60 & 15 & 398 & 425 \\
\hline $\mathrm{Sr}$ & 383.1 & 330.3 & 440.6 & 458.5 & 233.9 & 1159.9 & 17.1 & 223.5 & 13 & 79 & 4 & 4 & 177 & 188 \\
\hline V & 25 & 46 & 40 & 56 & 14 & 75 & 8 & 29 & 7 & 18 & 5 & 4 & 30 & 16 \\
\hline $\mathrm{Ni}$ & 2.7 & 3.7 & 2.2 & 6.8 & 1.5 & 3.8 & 0.5 & 1.8 & 5 & 2 & 5 & 5 & 12 & 5 \\
\hline $\mathrm{Zr}$ & 136.7 & 318.7 & 180.6 & 446.4 & 191.9 & 490.2 & 76.2 & 177.6 & 86 & 116 & 70 & 103 & 135 & 170 \\
\hline Sc & 3 & 5 & 3 & 4 & 2 & 4 & 3 & 4 & - & - & - & - & - & - \\
\hline $\mathrm{Cu}$ & 3.5 & 4.5 & 2.5 & 5.1 & 4.2 & 20 & 0.8 & 1.7 & 4 & 5 & 3 & 3 & 6 & 2 \\
\hline $\mathrm{Ce}$ & 62.5 & 161.4 & 70.6 & 131.1 & 116.2 & 311.4 & 33.3 & 122.9 & 21.13 & 48.41 & - & - & 21.21 & - \\
\hline Co & 3.6 & 6.3 & 6.1 & 8.1 & 2.8 & 8.1 & 1.7 & 4.1 & 77 & & - & 113 & 113 & 110 \\
\hline Dy & 1.26 & 4.45 & 1.2 & 2.73 & 1.46 & 2.77 & 5.58 & 3.96 & 4.5 & 5.26 & - & - & 1.01 & - \\
\hline Cs & 9.2 & 15 & 2.3 & 3.2 & 22 & 2.1 & 16.2 & 18.9 & - & - & - & - & - & - \\
\hline Er & 0.67 & 1.8 & 0.68 & 0.63 & 0.32 & 0.96 & 2.74 & 1.84 & 2.98 & 3.22 & - & - & 0.89 & - \\
\hline $\mathrm{Eu}$ & 0.74 & 1.78 & 0.72 & 2.43 & 1.19 & 2.25 & 0.14 & 1.2 & 0.29 & 0.87 & - & - & 1.04 & - \\
\hline $\mathrm{Ga}$ & 18.8 & 23.3 & 20.4 & 26.4 & 22.6 & 22 & 20 & 20.6 & & & - & - & - & - \\
\hline Gd & 2.28 & 8.1 & 1.98 & 7.09 & 4.59 & 7.6 & 4.15 & 6.15 & 3.82 & 5.46 & & & 2.13 & \\
\hline $\mathrm{Hf}$ & 4.2 & 9.5 & 5.1 & 11.9 & 6 & 11.8 & 2.9 & 6 & & & & & & \\
\hline Ho & 0.24 & 0.69 & 0.24 & 0.34 & 0.16 & 0.4 & 0.99 & 0.68 & 1.14 & 1.32 & & & 0.33 & \\
\hline $\mathrm{La}$ & 34.7 & 73.2 & 41.7 & 66.3 & 52.2 & 160.8 & 13.9 & 55.2 & 5.5 & 17.94 & & & 9.64 & \\
\hline $\mathrm{Lu}$ & 0.06 & 0.28 & 0.09 & 0.04 & 0.03 & 0.11 & 0.31 & 0.22 & 0.27 & 0.44 & & & 0.06 & \\
\hline $\mathrm{Nb}$ & 10.4 & 23 & 9.8 & 23.3 & 16 & 18.8 & 11.6 & 14.2 & 15 & 14 & 187 & 202 & 11 & 62 \\
\hline $\mathrm{Nd}$ & 21 & 72.2 & 22 & 61.2 & 51.5 & 109.4 & 14.2 & 53 & 10.7 & 23.92 & & & 8.93 & \\
\hline $\mathrm{Pr}$ & 6.65 & 18.87 & 6.97 & 15.81 & 13.31 & 31.51 & 4.07 & 14.16 & & & & & & \\
\hline Sm & 3.7 & 12.27 & 2.77 & 9.94 & 8.28 & 14.11 & 4.04 & 9.22 & 2.78 & 4.35 & & & 1.62 & \\
\hline Sn & 9 & 6 & 1 & 1 & 6 & 1 & 12 & 6 & & & & & & \\
\hline $\mathrm{Ta}$ & 1 & 2.5 & 0.8 & 1.1 & 1.7 & 0.8 & 2 & 1.7 & & & & & & \\
\hline $\mathrm{Tb}$ & 0.33 & 1.06 & 0.28 & 0.85 & 0.49 & 0.86 & 0.92 & 0.88 & & & & & & \\
\hline Th & 13.8 & 29.7 & 10.6 & 17.2 & 20.7 & 37.7 & 15.7 & 21.8 & & & & & & \\
\hline $\mathrm{Tm}$ & 0.09 & 0.31 & 0.1 & 0.07 & 0.04 & 0.14 & 0.42 & 0.28 & & & & & & \\
\hline $\mathrm{U}$ & 1.9 & 5.5 & 0.9 & 1.5 & 4.8 & 1.6 & 4.6 & 8.3 & & & & & & \\
\hline W & 0.5 & 1.1 & 0.5 & 0.5 & 0.7 & 0.5 & 6.9 & 1.9 & & & & & & \\
\hline $\mathrm{Y}$ & 8.2 & 23.8 & 8.2 & 15 & 6.1 & 13.9 & 32.9 & 23.3 & 28 & 31 & 38 & 43 & 4 & 15 \\
\hline $\mathrm{Yb}$ & 0.35 & 2.12 & 0.58 & 0.22 & 0.16 & 0.66 & 2.49 & 1.59 & & & & & 0.3 & \\
\hline$q$ & 26.98 & 30.79 & 29.63 & 26.53 & 30.56 & 25.7 & 42.9 & 30.76 & 37.44 & 41.38 & 29.8 & 27.69 & 38.73 & 29.96 \\
\hline or & 28.78 & 30.26 & 15.01 & 22.99 & 35.75 & 27.07 & 32.44 & 49.34 & 29.72 & 27.07 & 25.17 & 30.79 & 10.93 & 34.22 \\
\hline$a b$ & 30.8 & 21.49 & 34.95 & 26.32 & 23.27 & 25.72 & 16.67 & 10.58 & 23.02 & 20.56 & 40.06 & 35.13 & 31.82 & 28.85 \\
\hline an & 7.92 & 6.96 & 13.49 & 11.72 & 4.99 & 9.49 & 0.17 & 0 & 1.43 & 3.76 & 0 & 0 & 10.1 & 0.55 \\
\hline c & 0.87 & 2.54 & 0.1 & 1.83 & 1.25 & 1.77 & 3.89 & 3.56 & 1.39 & 1.86 & 0 & 0 & 0 & 0 \\
\hline $\mathrm{ac}$ & 0 & 0 & 0 & 0 & 0 & 0 & 0 & 0 & 0 & 0 & 0.56 & 3.18 & 0 & 0 \\
\hline $\mathrm{di}$ & 0 & 0 & 0 & 0 & 0 & 0 & 0 & 0 & 0 & 0 & 0.32 & 0.98 & 1.93 & 1.11 \\
\hline hy & 1.22 & 2.04 & 2.02 & 2.47 & 0.85 & 2.42 & 0.4 & 1.25 & 1.42 & 1.79 & 0 & 1.04 & 1.07 & 0.53 \\
\hline wo & 0 & 0 & 0 & 0 & 0 & 0 & 0 & 0 & 0 & 0 & 1.11 & 0.11 & 0 & 0 \\
\hline $\mathrm{mt}$ & 0 & 0 & 0 & 0 & 0 & 0 & 0 & 0 & 0 & 0 & 0.44 & 0 & 0 & 0 \\
\hline hem & 2.04 & 3.32 & 2.58 & 3.97 & 1.66 & 4.27 & 1.65 & 2.07 & 2.22 & 3.17 & 0.46 & 0 & 2.23 & 2.18 \\
\hline il & 0.13 & 0.11 & 0.13 & 0.06 & 0.09 & 0.11 & 0.04 & 0.09 & 0.11 & 0.11 & 0.09 & 0.11 & 0.04 & 0.26 \\
\hline ru & 0.22 & 0.72 & 0.37 & 1.4 & 0.29 & 1.04 & 0.06 & 0.34 & 0.13 & 0.34 & 0 & 0 & 0 & 0 \\
\hline tn & 0 & 0 & 0 & 0 & 0 & 0 & 0 & 0 & 0 & 0 & 0 & 0 & 0.68 & 0.26 \\
\hline ap & 0.32 & 0 & 0.23 & 0.93 & 0.32 & 0.79 & 0.12 & 0.74 & 0.6 & 0.23 & 0.07 & 0.07 & 0.14 & 0.28 \\
\hline Total & 99.28 & 98.79 & 98.51 & 98.21 & 99.02 & 98.37 & 98.34 & 98.6 & 97.48 & 100.3 & 98.1 & 99.27 & 97.67 & 98.2 \\
\hline
\end{tabular}


Table 3. Sm-Nd isotopic results

\begin{tabular}{l|c|c|c|c|c|c|c}
\hline Sample & $\mathbf{S m}$ & $\mathbf{N d}$ & ${ }^{143} \mathbf{N d} /{ }^{144} \mathbf{N d}$ & ${ }^{147} \mathbf{S m} /{ }^{144} \mathbf{N d}$ & $\varepsilon_{(\mathbf{0})}$ & $\varepsilon_{(\mathrm{T})}$ & $\mathbf{T}_{\mathrm{DM}}(\mathbf{G a})$ \\
\hline FB-15 & 1.563 & 6.105 & 0.512061 & 0.1548 & -11.25 & -10.545 & - \\
\hline FB-48 & 2.505 & 15.229 & 0.511962 & 0.0994 & -13.19 & -5.359 & 1.43 \\
\hline FB-86 & 2.837 & 20.085 & 0.511948 & 0.0854 & -13.46 & -2.218 & 1.29 \\
\hline FB-106 & 6.079 & 32.495 & 0.511922 & 0.1131 & -13.97 & -7.224 & 1.68 \\
\hline FB-108 & 5.956 & 35.607 & 0.511939 & 0.1011 & -13.63 & -5.909 & 1.48 \\
\hline FB-112 & 3.486 & 11.764 & 0.512176 & 0.1791 & -9.02 & -7.605 & - \\
\hline FB-113 & 1.119 & 3.003 & 0.512582 & 0.2252 & -1.10 & -3.980 & - \\
\hline FB-114 & 9.183 & 52.498 & 0.511805 & 0.1057 & -16.26 & -8.934 & 1.73 \\
\hline FB-116 & 3.019 & 16.333 & 0.511990 & 0.1117 & -12.64 & -5.728 & 1.56 \\
\hline AD-6 & 3.448 & 57.436 & 0.512211 & 0.0363 & -8.32 & 4.596 & 0.72 \\
\hline AD-12 & 8.975 & 50.78 & 0.511856 & 0.1068 & -15.0 & -8.027 & 1.68 \\
\hline AD-40 & 10.40 & 53.33 & 0.511716 & 0.1179 & -17.99 & -8.931 & 2.10 \\
\hline AD-13 & 5.282 & 24.523 & 0.512263 & 0.1302 & -7.31 & - & 1.41 \\
\hline AD-46 & 3.332 & 12.490 & 0.512341 & 0.1613 & -5.05 & - & - \\
\hline
\end{tabular}

Table 4. Representative results of zircon analyses from Quebra Anzol Granite

\begin{tabular}{l|c|c|c|c|c|c|c|c|c|c|c|c|c|c}
\hline Sample & $\mathbf{6 / 4}$ & $\mathbf{7 / 6}$ & $\mathbf{1 s}(\mathbf{\%})$ & $\mathbf{7 / 5}$ & $\mathbf{1 s}(\mathbf{\%})$ & $\mathbf{6 / 8}$ & $\mathbf{1 s}(\mathbf{\%})$ & Age 7/6 & $\mathbf{1 s ( M y )}$ & Age 7/5 & 1s(My) & Age 6/8 & 1s(My) & Rho \\
\hline 15-1-010-Z08 & 720 & 0.06802 & 3.6 & 1.2727 & 4.0 & 0.13570 & 1.7 & 869.1 & 72.9 & 833.6 & 22.5 & 820.3 & 13.6 & 0.67 \\
\hline 15-1-015-Z11 & 689 & 0.06877 & 1.4 & 1.3464 & 2.4 & 0.14199 & 1.9 & 891.8 & 27.7 & 866.0 & 13.7 & 855.9 & 15.5 & 0.81 \\
\hline 15-1-027-Z21 & 1422 & 0.06767 & 3.0 & 1.3195 & 4.4 & 0.14142 & 3.2 & 858.5 & 63.1 & 854.3 & 25.5 & 852.7 & 25.5 & 0.72 \\
\hline 15-1-026-Z20 & 9324 & 0.06567 & 1.1 & 1.2485 & 1.6 & 0.13788 & 1.1 & 796.0 & 23.2 & 822.7 & 8.8 & 832.7 & 8.6 & 0.86 \\
\hline 15-2-005-Z03 & 28151 & 0.06558 & 1.0 & 1.2648 & 1.4 & 0.13987 & 1.0 & 793.1 & 21.1 & 830.1 & 8.1 & 843.9 & 8.1 & 0.69 \\
\hline 15-2-013-Z09 & 5916 & 0.06657 & 0.6 & 1.2511 & 2.3 & 0.13631 & 2.2 & 824.3 & 12.3 & 823.9 & 13.0 & 823.7 & 17.2 & 0.97 \\
\hline 15-2-020-Z16 & 6703 & 0.06639 & 1.5 & 1.2531 & 2.0 & 0.13690 & 1.3 & 818.6 & 31.2 & 824.8 & 11.0 & 827.1 & 9.7 & 0.83 \\
\hline 15-2-027-Z21 & 930 & 0.06826 & 2.9 & 1.3003 & 3.1 & 0.13815 & 1.2 & 876.5 & 59.1 & 845.8 & 17.8 & 834.2 & 9.4 & 0.38 \\
\hline
\end{tabular}

Table 5. Representative results of zircon analyses from Monte Carmelo Granitic Complex

\begin{tabular}{|c|c|c|c|c|c|c|c|c|c|c|c|c|c|c|}
\hline Sample & $6 / 4$ & $7 / 6$ & (\%) & $7 / 5$ & $1 \mathrm{~s}(\%)$ & $6 / 8$ & $1 s(\%)$ & $\begin{array}{l}\text { Age } \\
7 / 6\end{array}$ & 1s(My) & $\begin{array}{l}\text { Age } \\
7 / 5\end{array}$ & $1 s(M y)$ & $\begin{array}{c}\text { Age } \\
6 / 8\end{array}$ & $1 \mathrm{~s}(\mathrm{My})$ & Rho \\
\hline \multicolumn{15}{|l|}{ FB 48} \\
\hline 22 Z14 & 3676 & 0.06154 & 1.1 & 0.7993 & 0.9 & 0.09420 & 0.6 & 658.0 & 23.1 & 596.4 & 4.0 & 80.3 & 3.4 & 0.62 \\
\hline $21 \mathrm{Z13}$ & 5593 & 0.06114 & 2.1 & 0.8031 & 1.7 & 0.09527 & 1.3 & 644.1 & 44.3 & 598.6 & 7.4 & 86.6 & .2 & 72 \\
\hline $09 \mathrm{Z3}$ & 100710 & 0.06079 & 2.1 & 0.8135 & 1.5 & 0.09706 & 1.4 & 631.7 & 44.2 & 604.4 & 0 & 97.2 & 0 & 90 \\
\hline $24 \mathrm{Z16}$ & 42852 & 0.06244 & 1.1 & 0.8222 & 1.0 & 0.09550 & 0.6 & 689.3 & 23.5 & 609.3 & 4.4 & 88.0 & .2 & .48 \\
\hline $11 \mathrm{Z5}$ & 8588 & 0.06427 & 3.2 & 0.8334 & 2.9 & 0.09405 & 1.2 & 750.4 & 65.8 & 615.5 & 13.5 & 579.4 & 7 & 0.36 \\
\hline $20 \mathrm{Z12}$ & 7229 & 0.06208 & 2.8 & 0.8438 & 2.5 & 0.09858 & 1.2 & 676.7 & 8.7 & 21.2 & 6 & 06.1 & 2 & 49 \\
\hline $25 \mathrm{Z17}$ & 1913 & 0.06397 & 4.3 & 0.8329 & 3.2 & 0.09442 & 2.8 & 740.7 & 87.6 & 615.2 & 14.8 & 581.6 & 15.5 & 0.86 \\
\hline $18 \mathrm{Z10}$ & 3920 & 0.06433 & 0.9 & 1.1237 & 1.4 & 0.12667 & 1.0 & 752.7 & 18.9 & 764.7 & & 58.9 & 5 & 74 \\
\hline $04 \mathrm{Z1C}$ & 52363 & 0.06462 & 0.7 & 1.1476 & 1.1 & 0.12879 & 0.9 & 762.1 & 14.8 & 776.1 & 2 & 781.0 & 6 & .73 \\
\hline $10 \mathrm{Z} 4$ & 42786 & 0.06428 & 1.1 & 40 & 2.7 & 0.13021 & 2.4 & 750.8 & 23.3 & \begin{tabular}{|l|}
779.1 \\
\end{tabular} & 14.6 & 89.1 & 18.2 & 91 \\
\hline $12 \mathrm{Z6}$ & 41781 & 0.06255 & 3.5 & 1.1588 & 3.9 & 0.13435 & 1.7 & 693.1 & 75.4 & \begin{tabular}{|l|}
781.4 \\
\end{tabular} & 21.5 & 812.6 & 13.2 & 0.43 \\
\hline $06 \mathrm{Z2C}$ & 38436 & 0.06587 & 1.0 & 1.1890 & 2.5 & 0.13091 & 2.3 & 802.3 & 21.3 & \begin{tabular}{|l|}
795.5 \\
\end{tabular} & 13.8 & 793.1 & 17.0 & 0.88 \\
\hline $15 \mathrm{Z9}$ & 17296 & 0.06611 & 2.9 & 1.1944 & 2.6 & 0.13103 & 1.1 & 809.9 & 58.6 & \begin{tabular}{|l|}
798.0 \\
\end{tabular} & 14.5 & 793.8 & 8.2 & 0.40 \\
\hline $13 \mathrm{Z7}$ & 2288 & 0.06933 & 1.5 & 1.2538 & 1.2 & 0.13118 & 0.8 & 908.4 & 30.1 & 825.1 & 6.8 & 794.6 & 6.2 & 0.64 \\
\hline $14 \mathrm{Z} 8$ & 38901 & 0.07241 & 4.0 & 319 & 3.7 & 841 & 1.5 & 997.6 & 79.4 & 881.3 & 21.6 & 835.6 & 11.9 & 39 \\
\hline $19 \mathrm{Z11}$ & 41225 & 0.08135 & 4.1 & 14742 & 71 & 0.13143 & 26 & 1230.0 & 775 & 919.9 & 101 & 796.0 & 97 & 0.86 \\
\hline \multicolumn{15}{|l|}{ FB 86} \\
\hline $08 \mathrm{Z4}$ & 241 & 0.06300 & 2.0 & 1.14317 & 2.1 & 0.13159 & 0.8 & 708.4 & 41.7 & \begin{tabular}{|l|}
774.0 \\
\end{tabular} & 11.6 & 797.0 & 3 & 0.34 \\
\hline $31 \mathrm{Z15}$ & 22079 & 0.06394 & 1.4 & 1.146681 & 1.7 & 0.13006 & 1.0 & 739.8 & 29.1 & 775.7 & 9.1 & 788.2 & 7.1 & 0.52 \\
\hline $40 \mathrm{Z2O}$ & 33759 & 0.06419 & 1.4 & 1.154574 & 1.6 & 0.13045 & 0.9 & 747.9 & 28.9 & \begin{tabular}{|l|}
779.4 \\
\end{tabular} & 8.8 & 790.4 & 6.5 & 0.45 \\
\hline $48 \mathrm{Z} 24$ & 36004 & 0.06525 & 1.4 & 1.193318 & 2.0 & 0.13264 & 1.5 & 782.4 & 28.4 & \begin{tabular}{|l|}
797.5 \\
\end{tabular} & 11.0 & 802.9 & 11.1 & 0.66 \\
\hline $03 \mathrm{Z1}$ & 17293 & 0.06853 & 1.8 & 1.213215 & 1.6 & 0.12840 & 0.9 & 884.6 & 37.3 & 806.7 & 8.7 & 778.7 & 6.8 & 0.52 \\
\hline $13 \mathrm{Z7}$ & 13538 & 0.06973 & 2.7 & 1.24786 & 2.5 & 0.12980 & 0.9 & 920.3 & 53.7 & 822.4 & 14.0 & 786.7 & 6.8 & 0.32 \\
\hline
\end{tabular}


Table 6. Representative results of zircon analyses from Perdizes Granite

\begin{tabular}{|c|c|c|c|c|c|c|c|c|c|c|c|c|c|c|}
\hline Sample & $6 / 4$ & $7 / 6$ & $1 s(\%)$ & $7 / 5$ & $1 s(\%)$ & $6 / 8$ & $1 s(\%)$ & $\begin{array}{c}\text { Age } \\
7 / 6\end{array}$ & $1 \mathrm{~s}(\mathrm{My})$ & $\begin{array}{c}\text { Age } \\
7 / 5\end{array}$ & 1s(My) & $\begin{array}{c}\text { Age } \\
6 / 8\end{array}$ & $1 \mathrm{~s}(\mathrm{My})$ & Rho \\
\hline $40 \mathrm{Z20}$ & 48875 & 0.05796 & 5.8 & 0.8244 & 5.9 & 0.10317 & 0.7 & 528.2 & 127.5 & 610.5 & 26.9 & 632.9 & 4.3 & 0.12 \\
\hline $08 \mathrm{Z4}$ & 70947 & 0.06034 & 3.2 & 0.8531 & 3.3 & 0.10255 & 0.7 & 615.7 & 69.4 & 626.4 & 15.3 & 629.3 & 4.0 & 0.20 \\
\hline $32 \mathrm{Z16}$ & 78955 & 0.05990 & 2.2 & 0.8589 & 2.4 & 0.10398 & 1.0 & 600.1 & 47.1 & 629.5 & 11.2 & 637.7 & 6.1 & 0.41 \\
\hline $04 \mathrm{Z2}$ & 49779 & 0.06138 & 1.3 & 0.8679 & 1.1 & 0.10255 & 0.8 & 652.5 & 27.8 & 634.4 & 5.0 & 629.4 & 4.5 & 0.59 \\
\hline $07 \mathrm{Z3}$ & 61956 & 0.06032 & 0.8 & 0.8680 & 2.1 & 0.10436 & 1.9 & 615.0 & 18.1 & 634.5 & 9.7 & 639.9 & 11.5 & 0.92 \\
\hline $12 \mathrm{Z6}$ & 38875 & 0.06151 & 1.1 & 0.8739 & 1.0 & 0.10305 & 0.6 & 656.9 & 24.2 & 637.7 & 4.6 & 632.3 & 3.6 & 0.52 \\
\hline 39 Z19 & 74074 & 0.06074 & 0.9 & 0.8867 & 1.0 & 0.10589 & 0.5 & 629.9 & 18.3 & 644.6 & 4.6 & 648.8 & 2.8 & 0.34 \\
\hline $47 \mathrm{Z23}$ & 108789 & 0.06097 & 0.6 & 0.8889 & 1.2 & 0.10573 & 1.0 & 638.3 & 12.2 & 645.8 & 5.7 & 647.9 & 6.4 & 0.79 \\
\hline $44 \mathrm{Z} 22$ & 54830 & 0.06145 & 1.1 & 0.8910 & 0.9 & 0.10516 & 0.7 & 655.1 & 23.9 & 646.9 & 4.3 & 644.6 & 4.1 & 0.66 \\
\hline $31 \mathrm{Z15}$ & 77481 & 0.06166 & 1.1 & 0.8913 & 0.9 & 0.10484 & 0.6 & 662.4 & 22.6 & 647.1 & 4.1 & 642.7 & 3.7 & 0.59 \\
\hline $20 \mathrm{Z10}$ & 50473 & 0.06212 & 1.4 & 0.8928 & 1.1 & 0.10424 & 0.9 & 678.3 & 30.5 & 647.9 & 5.4 & 639.2 & 5.5 & 0.72 \\
\hline $24 \mathrm{Z12}$ & 37472 & 0.06609 & 2.7 & 0.9480 & 2.5 & 0.10403 & 1.0 & 809.3 & 55.2 & 677.1 & 12.2 & 638.0 & 6.1 & 0.38 \\
\hline $43 \mathrm{Z} 21$ & 6690 & 0.07309 & 1.8 & 1.0482 & 1.7 & 0.10401 & 0.5 & 1016.4 & 35.3 & 728.0 & 8.7 & 637.9 & 3.1 & 0.21 \\
\hline
\end{tabular}

Table 7. Representative results of zircon analyses from Estrela do Sul Granite

\begin{tabular}{|c|c|c|c|c|c|c|c|c|c|c|c|c|c|c|}
\hline Sample & $6 / 4$ & $7 / 6$ & $1 s(\%)$ & $7 / 5$ & $1 \mathrm{~s}(\%)$ & $6 / 8$ & $1 s(\%)$ & $\begin{array}{c}\text { Age } \\
7 / 6 \\
\end{array}$ & 1s(My) & $\begin{array}{c}\text { Age } \\
7 / 5 \\
\end{array}$ & $1 \mathrm{~s}(\mathrm{My})$ & $\begin{array}{c}\text { Age } \\
6 / 8 \\
\end{array}$ & 1s(My) & Rho \\
\hline $06 \mathrm{Z9}$ & 616 & 0.06224 & 2.0 & 0.8313 & 1.5 & 0.09687 & 1.3 & 682.5 & 41.7 & 614.4 & 6.9 & 596.0 & - & .83 \\
\hline $11 \mathrm{Z6}$ & 5610 & 0.05958 & 0.8 & 0.8822 & 1.3 & 0.10738 & 1.0 & 588.4 & 16.4 & 642.1 & 6.1 & 657.5 & 6.5 & 0.78 \\
\hline $13 \mathrm{Z8}$ & 7028 & 0.06961 & 2.9 & 1.0472 & 2.3 & 0.10911 & 1.9 & 916.9 & 59.2 & 727.5 & 11.7 & 667.6 & 11.8 & 0.83 \\
\hline
\end{tabular}

Table 8. Representative results of zircon analyses from Cascalho Rico Granite

\begin{tabular}{|c|c|c|c|c|c|c|c|c|c|c|c|c|c|c|}
\hline Sample & $6 / 4$ & $7 / 6$ & (\%) & $7 / 5$ & s(\%) & $6 / 8$ & (0) & $\begin{array}{l}\text { Age } \\
7 / 6\end{array}$ & $1 \mathrm{~s}(\mathrm{My})$ & $\begin{array}{l}\text { Age } \\
7 / 5\end{array}$ & $1 \mathrm{~s}(\mathrm{My})$ & $\begin{array}{c}\text { Age } \\
6 / 8\end{array}$ & 1s(My) & Rho \\
\hline 9 Z19 & 138305 & 06089 & 262 & 35 & 240 & 0231 & 779 & 635.4 & 40.9 & 629.5 & 7.1 & 627.9 & 0 & 0.76 \\
\hline $44 \mathrm{ZZ2}$ & 111231 & 06099 & 3287 & 0.86334 & 1187 & 0.10266 & 7166 & 639.0 & 28.3 & 631.9 & 5.2 & 30.0 & 4.3 & 0.52 \\
\hline $45 \mathrm{Z23}$ & 80525 & 06095 & 4594 & 865517 & 2073 & 10299 & 8196 & 637.6 & 31.1 & 633.1 & .I & 31.9 & T.J & 0.59 \\
\hline $23 \mathrm{Z11}$ & 13721 & 0.06562 & 1.1190 & 1.188622 & 1.3274 & 0.13137 & 0.7142 & 794.4 & 23.5 & 795.3 & 7.3 & 995.7 & 5.3 & 0.41 \\
\hline $46 \mathrm{ZZ} 4$ & 16340 & 0.06534 & 97 & 1.20 & 87 & 34 & 31 & 785.4 & 25.8 & 801.2 & 0 & 306.9 & W & 0.40 \\
\hline $24 \mathrm{Z12}$ & 22582 & 0.06748 & 2.6745 & 1.213402 & 2.4226 & 0.13041 & 1.1322 & 852.7 & 54.6 & 806.8 & 13.4 & 790.2 & 8.4 & 0.43 \\
\hline $08 \mathrm{Z4}$ & 12979 & 0.06568 & 5.1510 & 1.213496 & 6.0181 & 0.13399 & 3.1120 & 796.3 & 108.0 & 806.8 & 33.5 & 810.6 & 23.7 & 0.51 \\
\hline $32 \mathrm{Z16}$ & 31901 & 0.06571 & 1.6334 & 1.220105 & 1.8506 & 0.13467 & 0.8699 & 797.1 & 34.2 & 809.8 & 10.3 & 814.5 & 6.7 & 0.35 \\
\hline $16 \mathrm{Z8}$ & 19084 & 0.06760 & 3.8498 & 1.238962 & 559 & 3293 & 28 & 856.3 & 0.0 & 818.4 & 17.0 & 804.6 & 16.7 & 0.66 \\
\hline $43 \mathrm{Z} 21$ & 19954 & 0.09719 & 3.3654 & 3.525266 & 2.4978 & 0.26308 & 2.2536 & 1570.8 & 61.7 & 1532.9 & 19.6 & 1505.6 & 30.2 & 0.91 \\
\hline $20 \mathrm{Z10}$ & 15192 & 0.09412 & 1.2828 & 3.534071 & 1.9659 & 0.27233 & 1.4897 & 1510.5 & 24.2 & 1534.9 & 15.6 & 1552.6 & 20.6 & 0.74 \\
\hline $04 \mathrm{Z2}$ & 27948 & 0.11475 & 1.6435 & 5.214779 & 1.3197 & 0.32960 & 0.9789 & 1875.9 & 29.3 & 1855.0 & 11.2 & 1836.4 & 15.6 & 0.70 \\
\hline $35 \mathrm{Z17}$ & 16142 & 0.12280 & 1.7893 & 6.131792 & 1.5301 & 0.36215 & 0.9267 & 1997.3 & 31.5 & 1994.8 & 13.3 & 1992.4 & 15.9 & 0.52 \\
\hline $12 \mathrm{Z6}$ & 28341 & 0.12184 & 0.8823 & 6.858978 & 1.7656 & 0.40828 & 1.5294 & 1983.4 & 15.7 & 2093.4 & 15.6 & 2207.1 & 28.6 & 0.87 \\
\hline
\end{tabular}

Table 9. Representative results of zircon analyses from Galheirinho Granite

\begin{tabular}{|c|c|c|c|c|c|c|c|c|c|c|c|c|c|c|}
\hline Sample & $6 / 4$ & $7 / 6$ & $1 \mathrm{~s}(\%)$ & $7 / 5$ & $1 \mathrm{~s}(\%)$ & $6 / 8$ & $1 s(\%)$ & $\begin{array}{c}\text { Age } \\
7 / 6 \\
\end{array}$ & 1s(My) & $\begin{array}{c}\text { Age } \\
7 / 5 \\
\end{array}$ & 1s(My) & $\begin{array}{c}\text { Age } \\
6 / 8\end{array}$ & 1s(My) & Rho \\
\hline $24 \mathrm{Z12}$ & 9441 & 05754 & 2.7 & 0.8561 & 2.9 & 0.10790 & 1.0 & 512.3 & 59.7 & 628.0 & 13.6 & 660.6 & 6.3 & 0.34 \\
\hline $5 \mathrm{Z7}$ & 318 & 06073 & 0.7 & 0.8595 & . & 0.10264 & 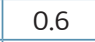 & 629.8 & 5.6 & 629.8 & 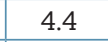 & 29.8 & . & 0.56 \\
\hline $19 \mathrm{Z9}$ & 24185 & 0.06013 & 0.8 & 0.8639 & 1.2 & 0.10420 & 0.9 & 608.2 & 17.2 & 632.3 & 5.5 & 639.0 & 5.2 & 0.72 \\
\hline & & 0.060 & 0.8 & 0.8844 & 1.1 & & 0. & 5.8 & 8.3 & 643.4 & 0 & 651.3 & .J. & 0.52 \\
\hline $23 \mathrm{Z11}$ & 537 & 0.05982 & 0.7 & 0.8855 & 1.0 & 0. & 0.7 & 597.0 & 5.3 & 644.0 & 47 & 657.4 & 44 & 0.66 \\
\hline $27 \mathrm{Z13}$ & 30 & 0.06070 & 0.7 & 0. & 1.1 & 0 & 0. & 628.6 & - & 654.3 & & 661.8 & 5.2 & 0.73 \\
\hline $07 \mathrm{Z3}$ & 22394 & 0.06425 & 3.5 & 0.9907 & 3.0 & 0.11183 & 1.9 & 749.8 & 72.9 & 699.1 & 15.0 & 683.4 & 12.1 & 0.61 \\
\hline $08 \mathrm{Z4}$ & 77375 & 0.06293 & 1.2 & 1.0546 & 3.4 & 0.12155 & 3.2 & 705.8 & 26.2 & 731.2 & 17.9 & 739.5 & 22.4 & 0.92 \\
\hline
\end{tabular}


Table 10. Representative results of zircon analyses from Pirapetinga Granite

\begin{tabular}{|c|c|c|c|c|c|c|c|c|c|c|c|c|c|c|}
\hline Sample & $6 / 4$ & $7 / 6$ & $1 \mathrm{~s}(\%)$ & $7 / 5$ & (\%) & $6 / 8$ & (\%) & $\begin{array}{l}\text { Age } \\
7 / 6\end{array}$ & $1 \mathrm{~s}(\mathrm{My})$ & $\begin{array}{c}\text { Age } \\
7 / 5\end{array}$ & 1s(My) & $\begin{array}{c}\text { Age } \\
6 / 8\end{array}$ & $1 \mathrm{~s}(\mathrm{My})$ & Rh \\
\hline $25 \mathrm{Z14}$ & 3057 & 0.06451 & 0.7 & 0.2088 & 2.4 & 0.02347 & 2.3 & 758.5 & 14.5 & 192.5 & 4.2 & 149.6 & 3.4 & 0.96 \\
\hline $47 \mathrm{Z25}$ & 12877 & 0.07352 & 0.7 & 0.4180 & 4.0 & 0.04124 & 4.0 & 1028.2 & 13.5 & 354.6 & 12.1 & 260.5 & 10.2 & 0.99 \\
\hline $23 \mathrm{Z12}$ & 303 & 0.14080 & 1.6 & 0.4234 & 3.6 & 0.02181 & 3.3 & 2237.1 & 27.6 & 358.5 & 11.0 & 139.1 & 4.5 & 0.90 \\
\hline 32 Z19 & 65157 & 0.06610 & 0.7 & 0.4524 & 9.7 & 0.04964 & 9.6 & 809.5 & 14.5 & 379.0 & 30.6 & 312.3 & 29.4 & 1.00 \\
\hline 39Z21B & 17933 & 0.07300 & 1.8 & 0.4954 & 2.8 & 0.04922 & 2.2 & 1013.9 & 37.0 & 408.6 & 9.5 & 309.7 & 6.5 & 0.76 \\
\hline $07 \mathrm{Z3}$ & 20613 & 0.06836 & 0.5 & 0.5128 & 4.4 & 0.05441 & 4.3 & 879.5 & 10.7 & 420.4 & .0 & 341.5 & 1.4 & .00 \\
\hline $12 \mathrm{Z6}$ & 578 & 0.07125 & 1.9 & 0.5143 & 8.2 & 0.05235 & 8.0 & 964.6 & 39.5 & 421.3 & 28.4 & 328.9 & 25.7 & 0.97 \\
\hline $48 \mathrm{Z} 26$ & 54085 & 0.06742 & 1.3 & 0.8504 & 5.0 & 0.09149 & 4.8 & 850.6 & 26.4 & 624.9 & 23.2 & 564.3 & 26.0 & 0.96 \\
\hline $19 \mathrm{Z10}$ & 344898 & 0.15969 & 3.5 & 0.8685 & 11.7 & 0.03945 & 11.2 & 2452.3 & 58.7 & 634.8 & 55.4 & 249.4 & 27.4 & 0.96 \\
\hline $14 \mathrm{Z8}$ & 45750 & 0.07420 & 0.8 & 0.8935 & 3.5 & 0.08733 & 3.4 & 1047.0 & 15.8 & 648.3 & 16.8 & 539.7 & 17.7 & 0.97 \\
\hline 46 Z24 & 128630 & 0.06 & 0.6 & 0.9635 & 1.6 & 0.10488 & 1.5 & 826.1 & 12.2 & 685.1 & 7.9 & 42.9 & 9.0 & .94 \\
\hline $13 \mathrm{Z7}$ & 29856 & 0.06771 & 0.7 & 0.9763 & 2.6 & 0.10458 & 2.6 & 859.6 & 14.3 & 691.7 & 13.3 & 641.2 & 15.6 & 0.97 \\
\hline $24 \mathrm{Z13}$ & 380121 & 0.07402 & 0.5 & 0.9814 & 1.1 & 0.09616 & 1.0 & 1042.1 & 11.1 & 694.3 & 5.6 & 591.9 & J. & .86 \\
\hline $40 Z 21 \mathrm{~N}$ & 120951 & 0.06330 & 0.6 & 0.9951 & 1.5 & 0.11402 & 1.4 & 718.2 & 13.0 & 701.3 & 7.6 & 696.1 & 9.0 & 0.93 \\
\hline $41 \mathrm{Z22B}$ & 89110 & 0.07946 & 1.4 & 0.9990 & 2.0 & 0.09119 & 1.5 & 1183.5 & 27.6 & 703.3 & 10.2 & 562.6 & 7.8 & 0.72 \\
\hline $42 \mathrm{Z} 22 \mathrm{~N}$ & 89357 & 0.06676 & 0.6 & 1.0043 & 1.8 & 0.10910 & 1.6 & 830.4 & 13.4 & 706.0 & 8.9 & 667.5 & 10.3 & 0.91 \\
\hline $05 \mathrm{Z1}$ & 75737 & 0.06533 & 0.6 & 1.0087 & 1.3 & 0.11198 & 1.2 & 785.0 & 11.6 & 708.2 & 6.7 & 684.2 & 7.7 & 0.92 \\
\hline $45 \mathrm{Z23}$ & 295659 & 0.10013 & 1.5 & 1.0095 & 3.2 & 0.07312 & 2.8 & 1626.6 & 27.5 & 708.6 & 16.1 & 454.9 & 12.2 & 0.89 \\
\hline $06 \mathrm{Z2}$ & 237508 & 0.06814 & 0.5 & 1.0224 & 0.9 & 0.10882 & 0.8 & 872.9 & 9.9 & 715.1 & 4.8 & 665.9 & 5.1 & 0.86 \\
\hline $11 \mathrm{Z5}$ & 267655 & 0.08099 & 1.1 & 1.0769 & 2.9 & 0.09644 & 2.7 & 1221.3 & 21.6 & 742.1 & 15.4 & 593.5 & 15.4 & 0.93 \\
\hline $30 \mathrm{Z17}$ & 68643 & 0.06707 & 3.5 & 1.0840 & 5.5 & 0.11722 & 4.2 & 839.8 & 73.9 & 745.6 & 28.9 & 714.6 & 28.1 & 0.76 \\
\hline $08 \mathrm{Z4}$ & 93332 & 0.06649 & 0.8 & 1.1114 & 2.3 & 0.12123 & 2.2 & 821.8 & 16.1 & 758.9 & 12.5 & 737.7 & 15.4 & 0.93 \\
\hline $26 \mathrm{Z15}$ & 3893 & 0.08077 & 2.2 & 1.1268 & 3.7 & 0.10118 & 2.9 & 1215.8 & 43.3 & 766.2 & 19.8 & 621.3 & 17.4 & 0.79 \\
\hline $31 \mathrm{Z18}$ & 83813 & 0.06590 & 0.5 & 1.1313 & 1.7 & 0.12452 & 1.6 & 803.1 & 11.3 & 768.4 & 9.1 & 756.5 & 11.4 & 0.96 \\
\hline $20 \mathrm{Z11}$ & 52554 & 0.06977 & 0.9 & 1.1586 & 2.4 & 0.12044 & 2.3 & 921.7 & 19.0 & 781.3 & 13.3 & 733.1 & 15.7 & 0.92 \\
\hline 29 Z16 & 59290 & 0.06477 & 0.6 & 1.1704 & 1.5 & 0.13106 & 1.4 & 766.8 & 12.0 & 786.8 & 8.2 & 793.9 & 10.3 & 0.93 \\
\hline $18 \mathrm{Z9}$ & 150863 & 0.06477 & 0.6 & 1.2635 & 1.7 & 0.14148 & 1.6 & 766.9 & 12.8 & 829.5 & 9.4 & 853.0 & 12.4 & 0.94 \\
\hline
\end{tabular}

equigranular, fine to medium light grey and slightly deformed, consisting of quartz, orthoclase, plagioclase, biotite, titanite, apatite, and zircon with epidote and sericite as secondary minerals formed over feldspars. This fact indicates hydrothermal alteration. They are classified as tonalite and granodiorite.

However, in the FB-48 and FB-60 samples, the granites have porphyritic and equigranular textures and besides the mentioned minerals, they also present muscovite and garnet being classified as syenogranite and monzogranite respectively (Fig. 4). Chemically, the two groups have notable differences, being more evident the high levels of $\mathrm{TiO}_{2}, \mathrm{CaO}, \mathrm{MgO}, \mathrm{Ni}$ and $\mathrm{Zr}$ in the FB-98 sample and high $\mathrm{CaO}$ and $\mathrm{MgO}$ in the FB-86 (Figs. 5 and 6). $\mathrm{Ta}$ and $\mathrm{Rb}$ are high in FB-60 and $\mathrm{Cr}$ in the FB-48. All present normative corundum that is lower in the FB-86, whose alumina saturation index is of 0.99 being, therefore, metaluminous, and the others peraluminous (Fig. 8). The $\mathrm{Na}_{2} \mathrm{O} / \mathrm{K}_{2} \mathrm{O}$ ratios are higher than one in FB-86, and smaller than one in the others. In the AnxAbxOr diagram they are classified as granodiorite and granite (Fig. 7). The LREE have a high fractionation compared to HREE $\left(\mathrm{La}_{\mathrm{N}} / \mathrm{Yb}_{\mathrm{N}}=67.02\right.$ and 203.68) in the samples FB-86 and

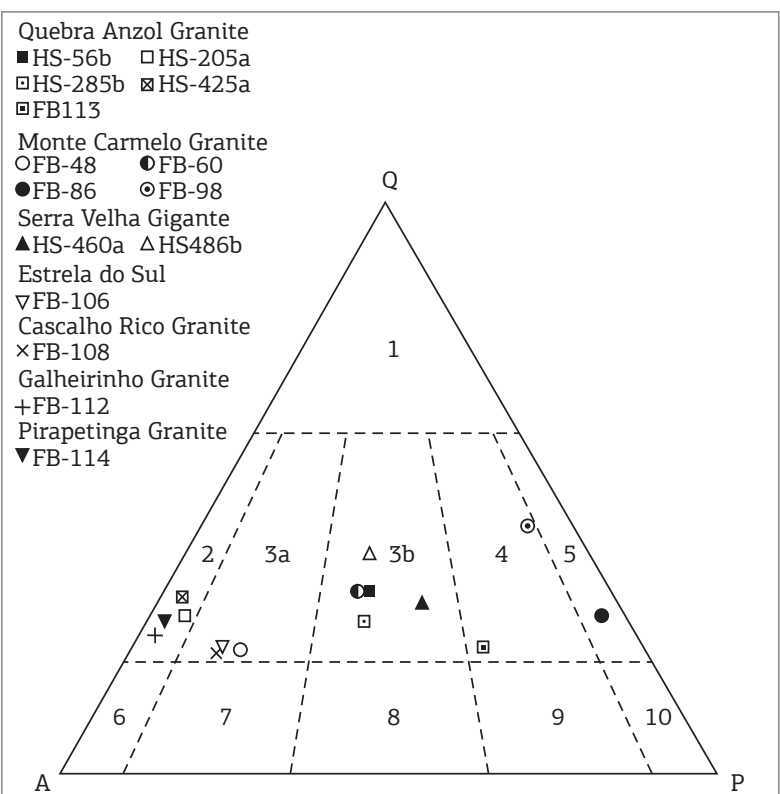

Figure 4. Modal QAP diagram for granitoids from Araxá Group.

FB-98, and lower ones in FB-60 and FB-48 $\left(\mathrm{La}_{\mathrm{N}} / \mathrm{Yb}_{\mathrm{N}}=\right.$ 23.28 to 48.56$)$. The $\mathrm{Eu} / \mathrm{Eu} *$ ratios range from 0.88 to 


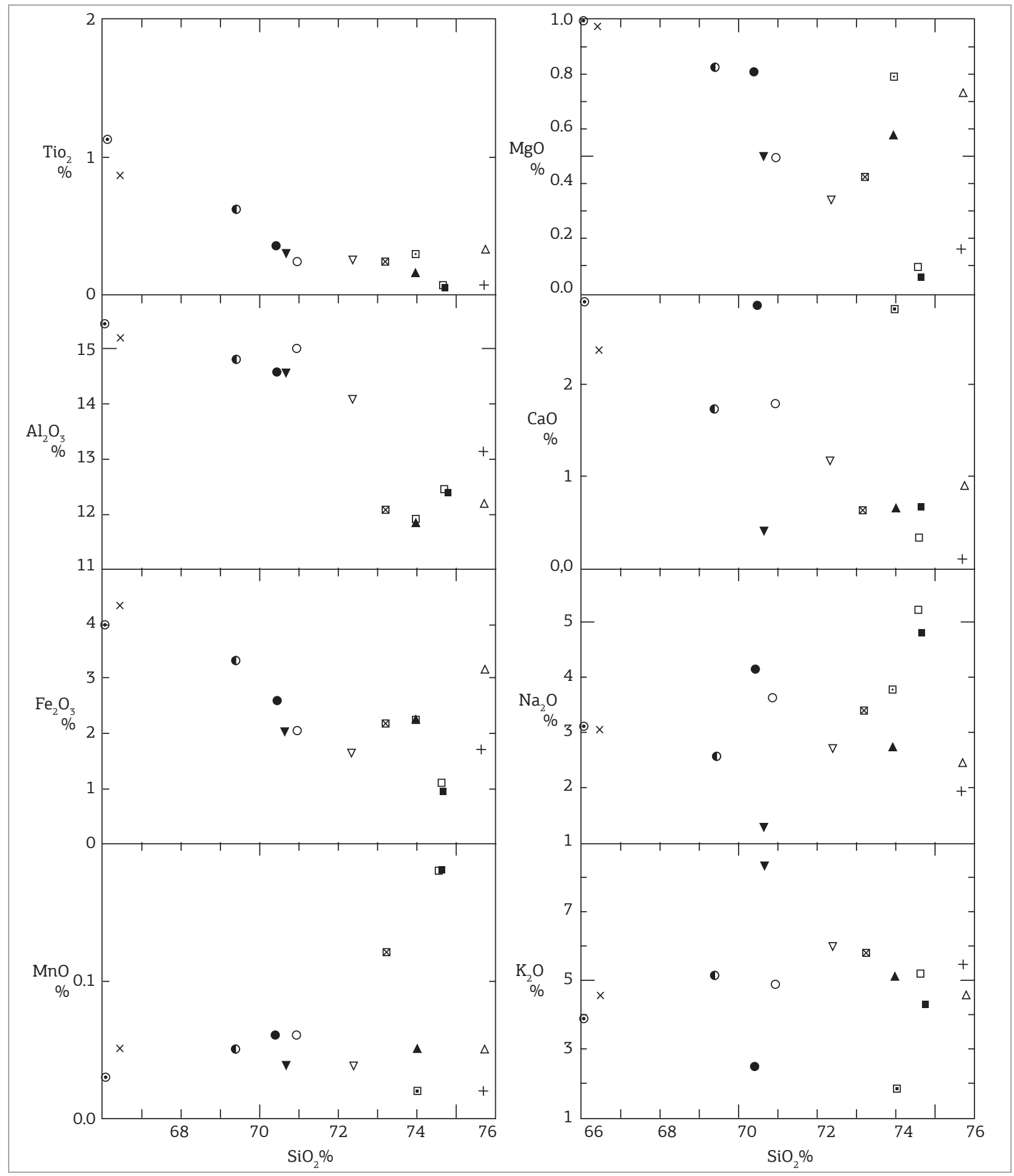

Figure 5. Bivariate oxide-oxide major element plots for the granitoids from Araxá Group. Simbology at Fig. 4.

0.94 in FB-98 and FB-86, and from 0.54 to 0.78 in FB60 and FB-48. Therefore, only the FB-60 sample presents slight negative Eu anomaly (Fig. 9). FB-98 stands out with the highest fractions of LREE in relation to HREE. In the multi-element diagram, all samples have subduction zone component (negative anomalies of $\mathrm{Nb}$ and $\mathrm{Ta}$ ) and present negative anomalies of $\mathrm{Sr}, \mathrm{P}$ and Ti (Fig. 10). The similarity between the curves in the FB-48 and FB-86 samples is remarkable, and they plot in the field of collisional granites in the R1xR2 diagram (Fig. 11); collisional granites and volcanic arc in the $\mathrm{NbxY}$ (Fig. 12); collisional granite and volcanic arc in the $\mathrm{RbxY}+\mathrm{Nb}$ (Fig. 14); and volcanic arc 


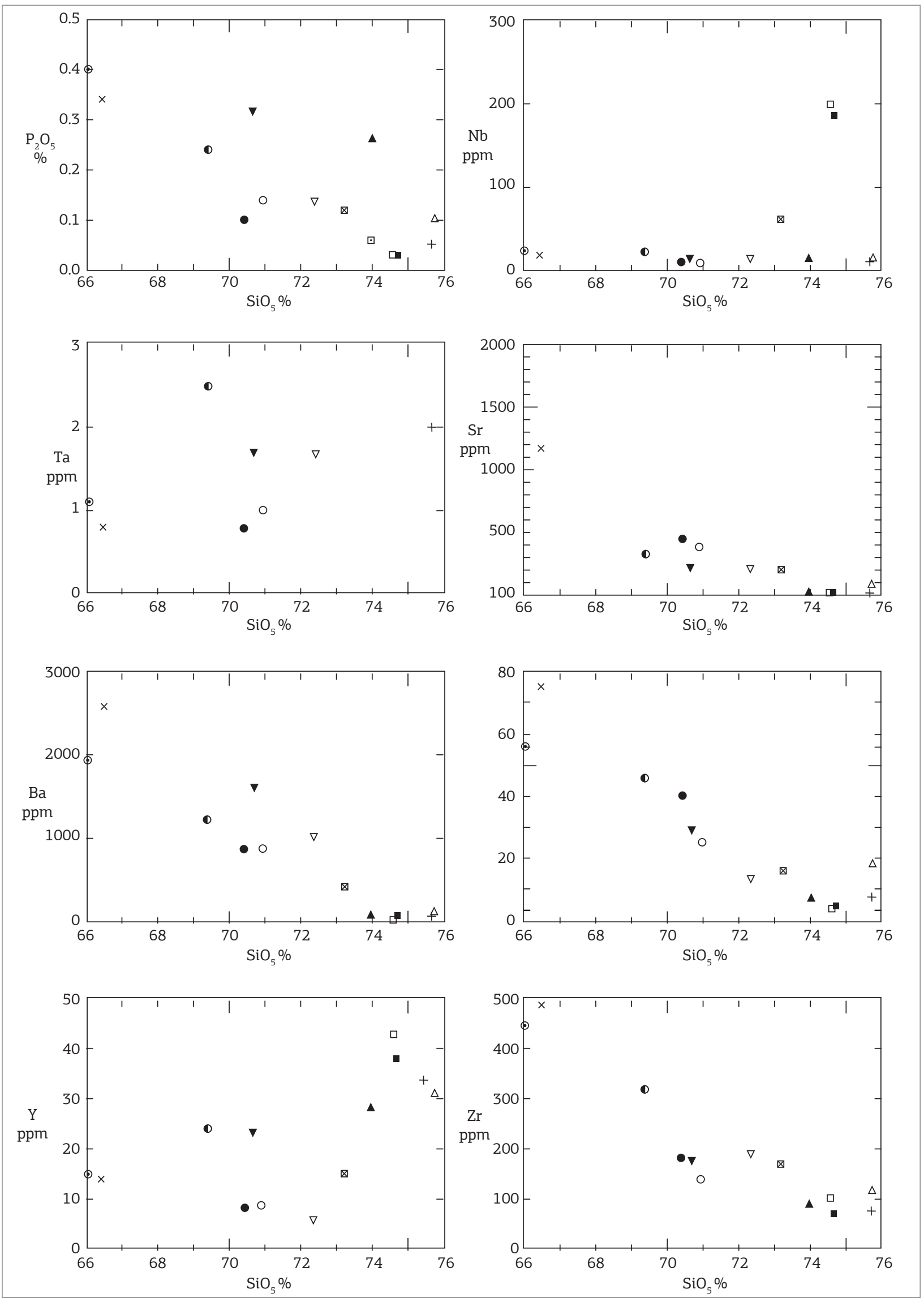

Figure 6. Bivariate oxide-oxide minor and trace element plots for the granitoids from Araxá Group. Simbology at Fig. 4. 


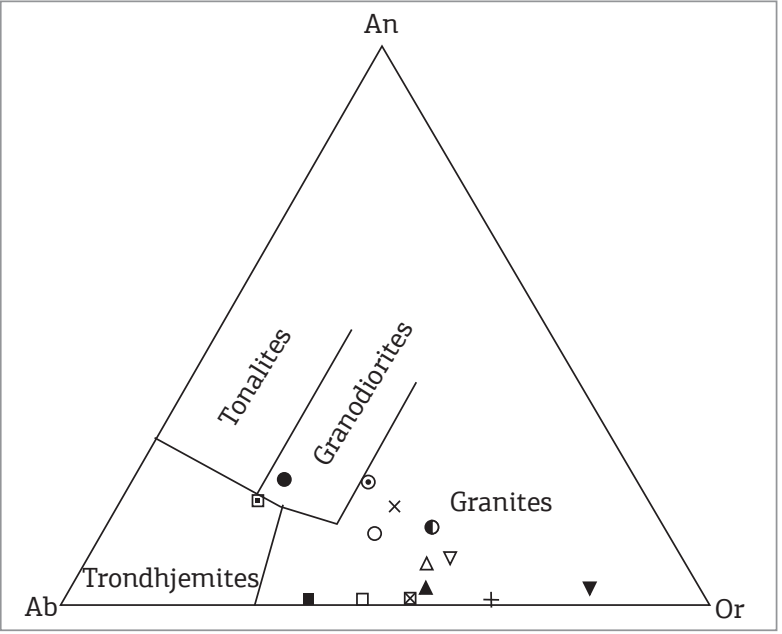

Figure 7. Or:Ab:Na diagram for Araxá Group granitoids (adapted from Barker 1979 apud Rollinson 1993). Simbology at Fig. 4.

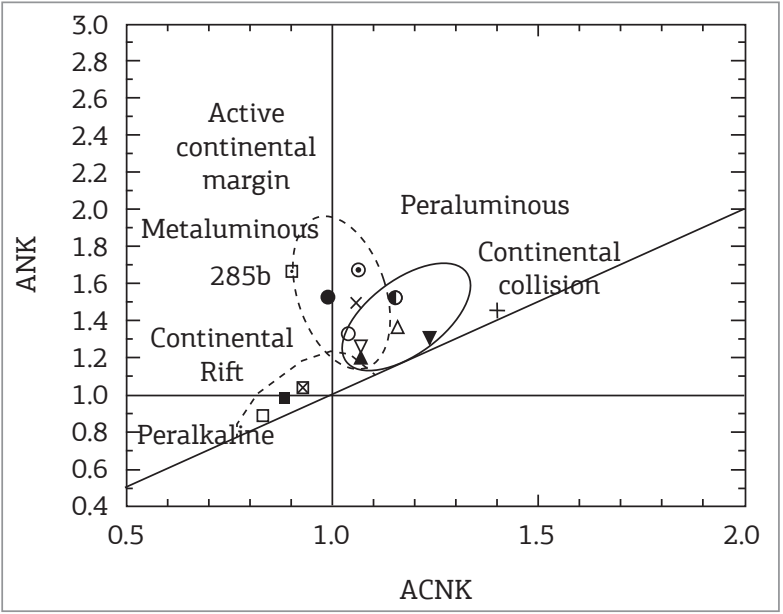

Figure 8. Shand Index (Maniar \& Piccoli 1989) for granitoids from Araxá Group. Simbology at Fig. 4.

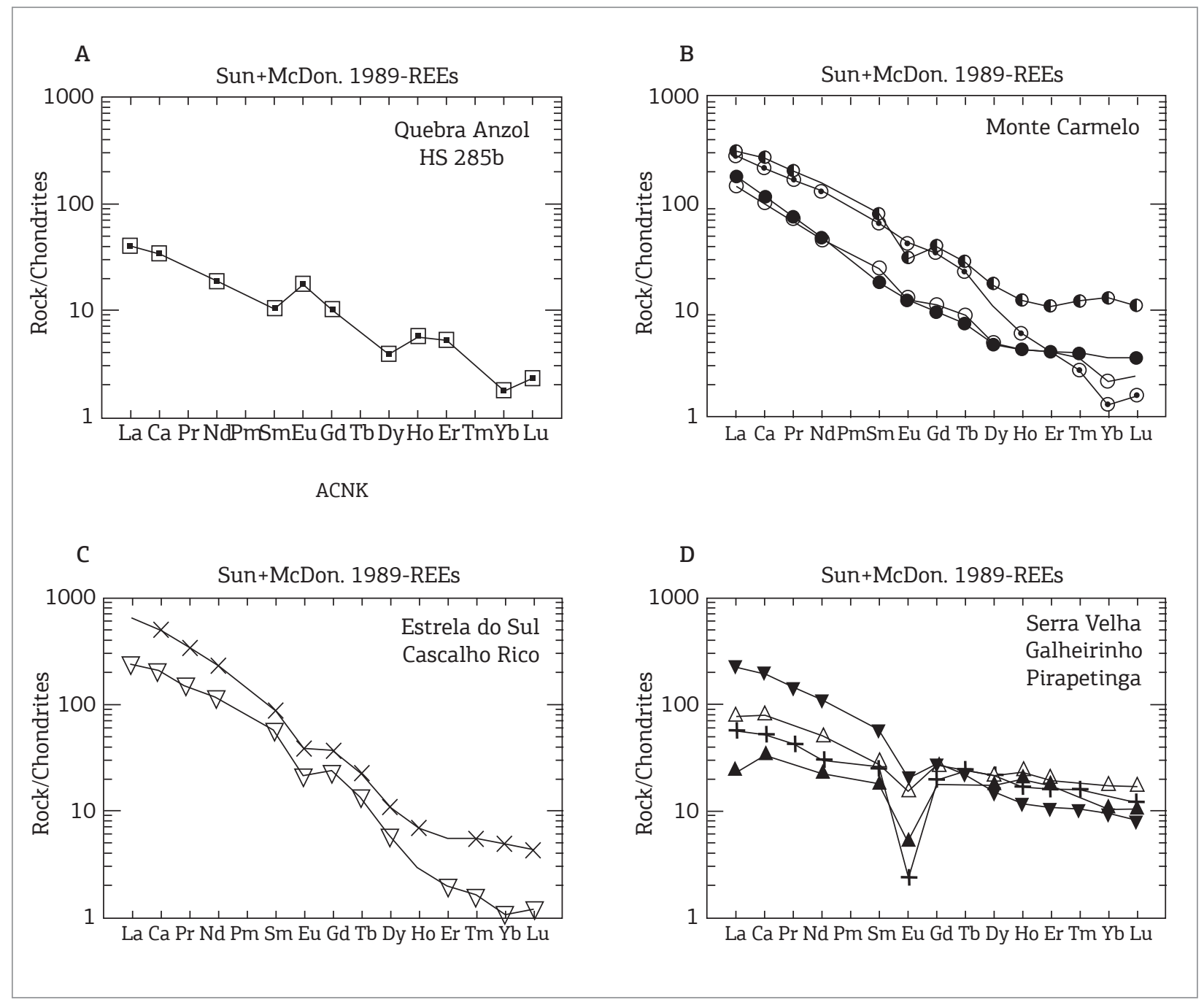

Figure 9. Rare earth elements patterns for Araxá Group granitoids. Normalization based on Sun and McDonough (1989) apud Rollinson (1993). Simbology at Fig. 4. 


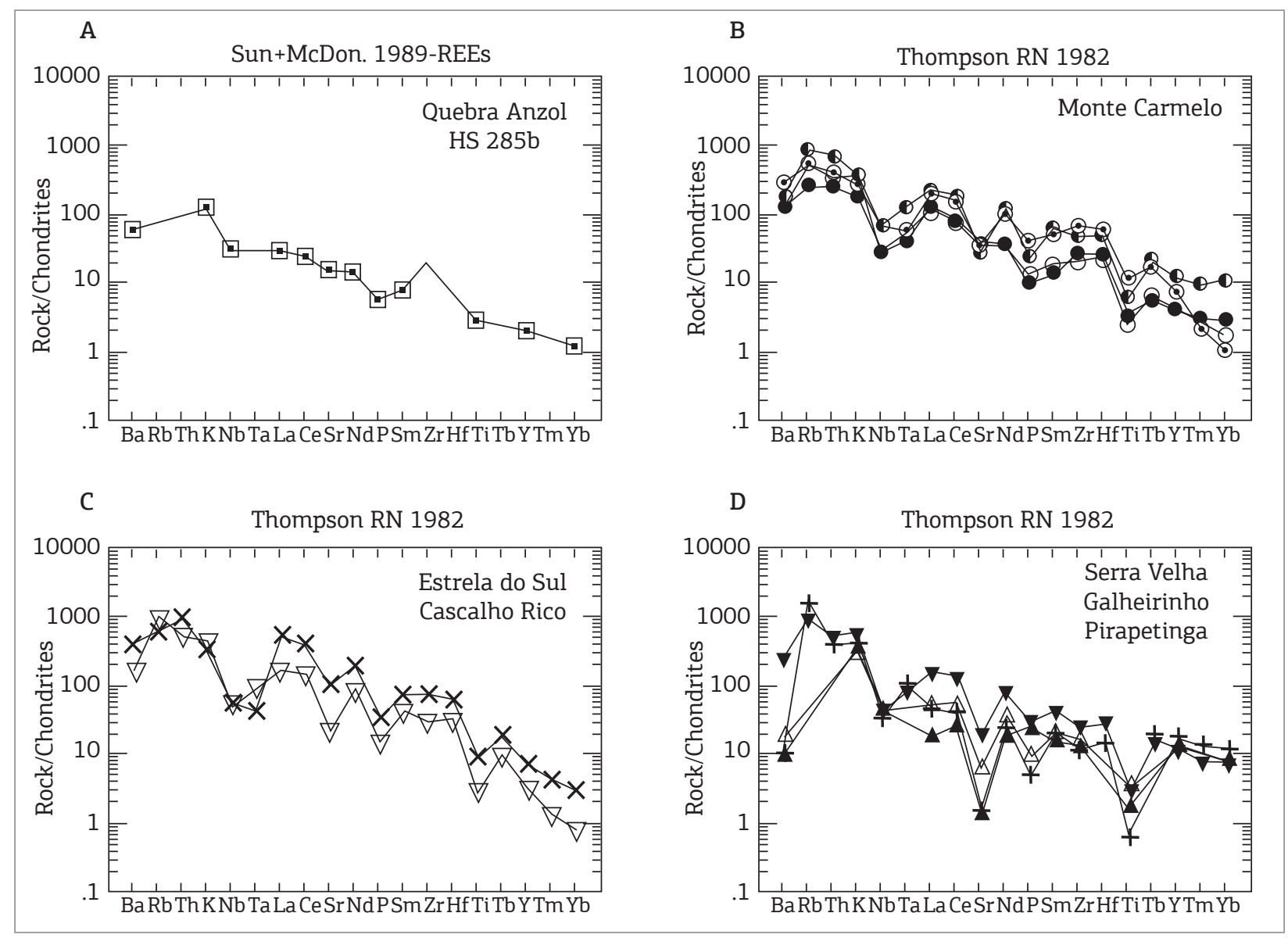

Figure 10. Spider diagrams for Araxá Group granitoids. Normalization based on Thompson (1982) apud Rollinson (1993). Simbology at Fig. 4.

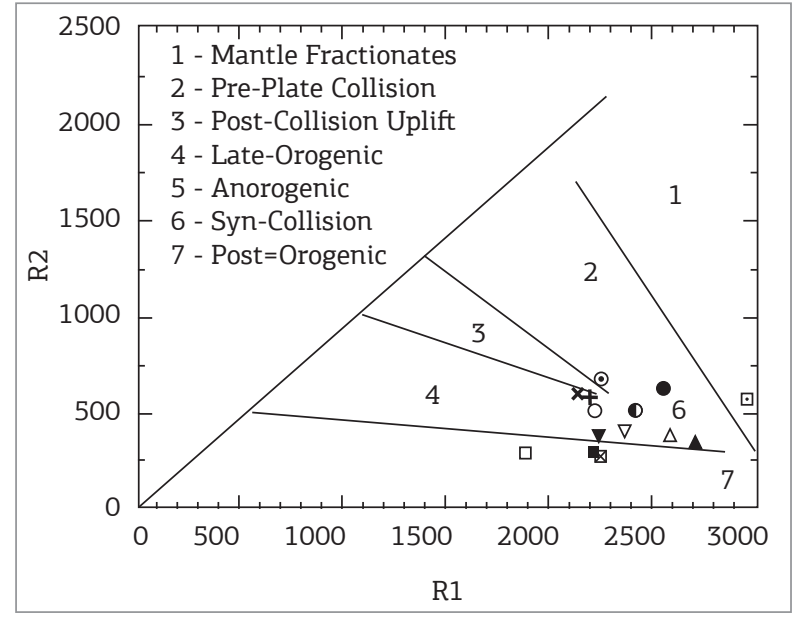

Figure 11. R1xR2 diagram (Batchelor \& Bowden 1985) for Araxá Group granitoids. Simbology at Fig. 4.

granites in the HfxRb/30x3Ta one (Fig. 15). They are similar to the I and III granite types in the $\mathrm{Rb} / \mathrm{ZrxSiO}_{2}$ and TaxNb diagrams (Figs. 16 and 17).

The FB-60 sample plots in the transition to the second granites type. They are, therefore, collisional granites

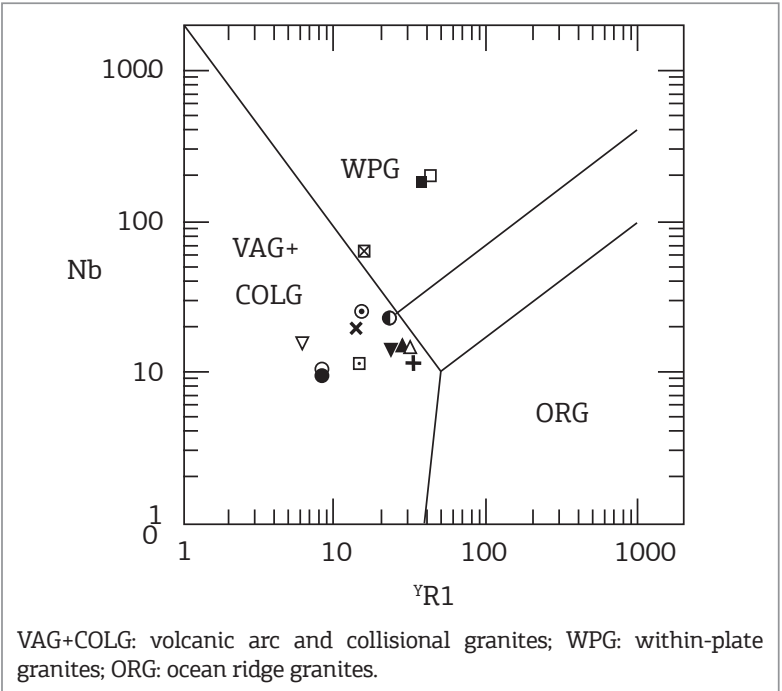

Figure 12. NbxY tectonic discriminant diagram (Pearce et al. 1984) for some Araxá Group granitoids. Simbology at Fig. 4.

originated by the melting of volcanic arc rocks. FB-86 and FB-98 samples instead suggest an origin in the pre-collisional volcanic arc as shown in the mentioned diagrams. 

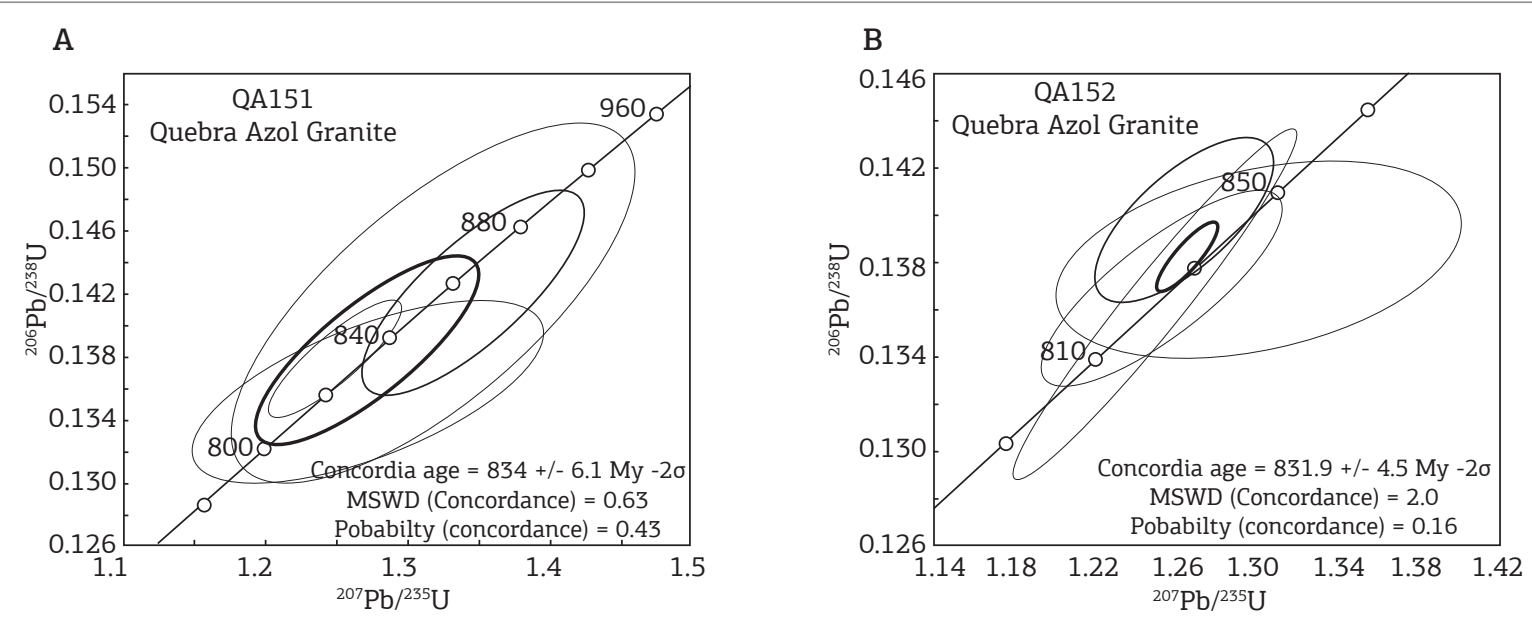

VAG: volcanic arc granites; Syn-COLG: syn-collisional granites; WPG: within plate granites; ORG: ocean ridge granites.

Figure 13. RbxY + Nb tectonic discriminant diagram (Pearce et al. 1984) for some Araxá Group granitoids. Simbology as Fig. 4.

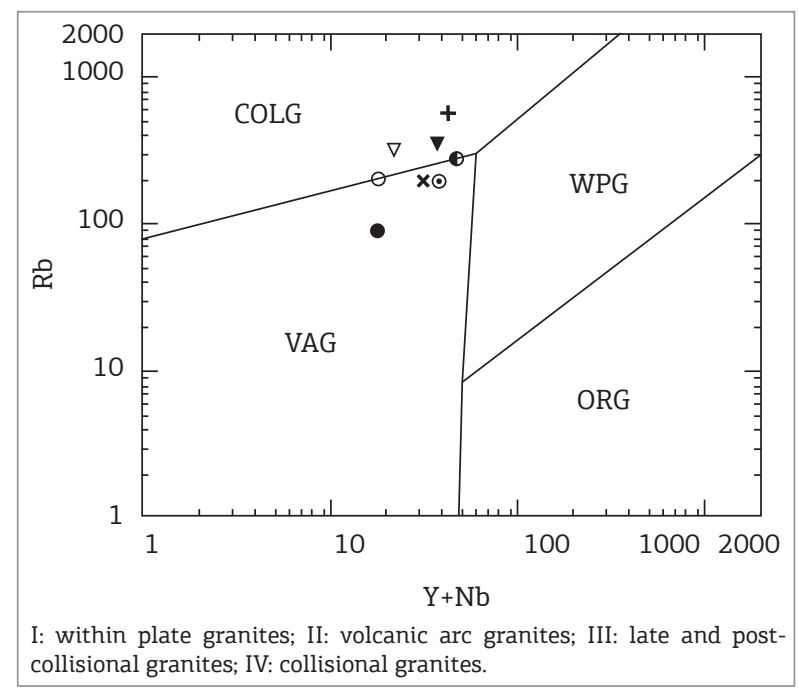

Figure 14. HfxRb/30x3Ta tectonic discriminant diagram (Harris et al. 1986) for some Araxá Group granitoids. Simbology at Fig. 4.

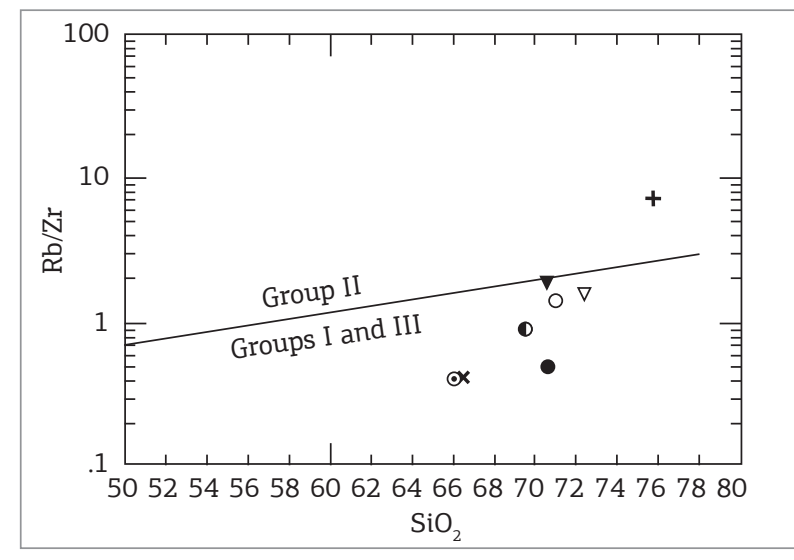

Figure 16. TaxNb tectonic discriminant diagram (Harris et al. 1986) for some Araxá Group granitoids. Simbology as Fig. 4 and fields like Fig. 14.

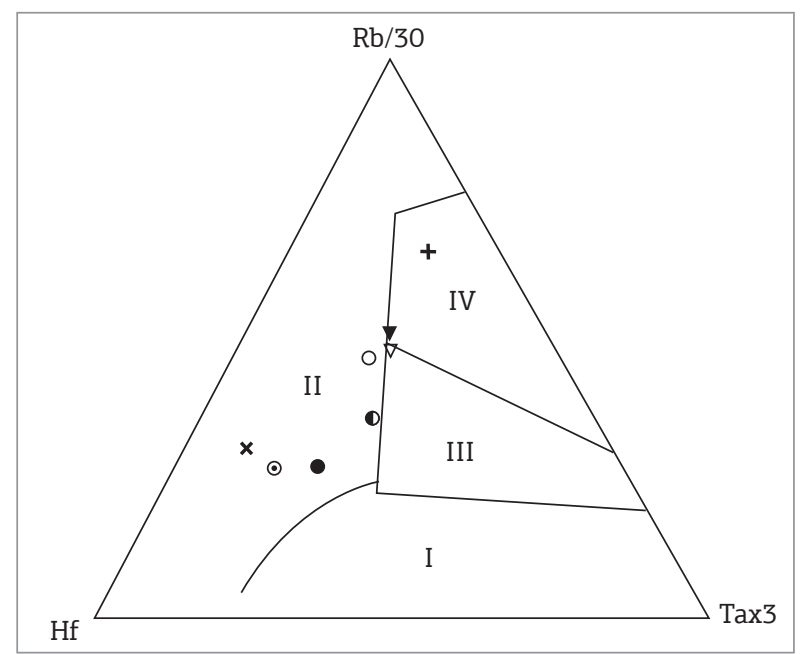

Figure $15 . \mathrm{Rb} / \mathrm{ZrxSiO}_{2}$ tectonic discriminant diagram (Harris et al. 1986) for some Araxá Group granitoids. Simbology at Fig. 4 and fields like Fig. 14.

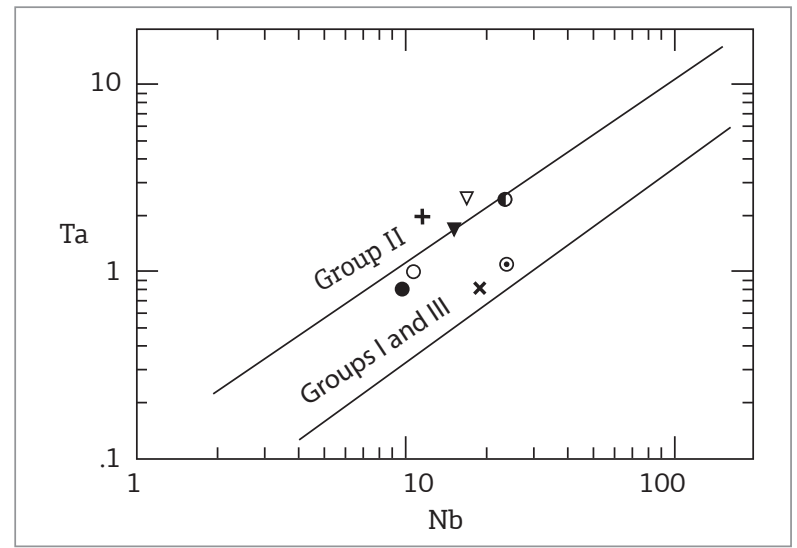

Figure 17. Concordia diagram for Quebra Anzol Granite. 
The FB-48 sample has zircon grains with much loss of lead, probably that happened during the transport of nappes. Nevertheless, it is possible to identify two populations. The most reliable result is the age of $6 / 8$ of $586 \pm 22 \mathrm{Ma}$ and inheritance nearly $790 \mathrm{Ma}$ (Fig. 18). The crystallization age should be around $630 \mathrm{Ma}$, and the inheritance may be from the granites of $790 \mathrm{Ma}$. Its $\varepsilon_{\mathrm{Nd}(630)}$ is of -5.359 and its $\mathrm{T}_{\mathrm{DM}}$ of $1.43 \mathrm{Ga}$, indicating reworking of crustal sources. The FB-86 sample seems to have incorporation of lead, as the majority (20 out of 26) of the grains present high rations of ${ }^{206} \mathrm{~Pb} /{ }^{204} \mathrm{~Pb}$, not being useful for the age calculation. Other six grains are of about $790 \mathrm{Ma}$, by Tera-Wasserburg's method (Fig. 19), and the average is of 6/8, which is interpreted as the time of body crystallization. Its $\varepsilon_{\mathrm{Nd}(790)}$ is -2.218 and its $\mathrm{T}_{\mathrm{DM}}$ is $1.29 \mathrm{Ga}$. This shows an origin from juvenile sources at the end of the Mesoproterozoic period. In the $\varepsilon_{\mathrm{Nd}(790)} \times \mathrm{T}$ diagram (Fig. 20), the evolution curve of $\mathrm{Nd}$ is similar to the granites of the Goiás Magmatic Arc. Two samples were investigated for Sm-Nd: AD-06 is a porphyritic light grey, slightly deformed granite, with K-feldspar, quartz and biotite, with $\varepsilon_{\mathrm{Nd}(630)}$ of 4.596 and $0.72 \mathrm{Ga}$ for $\mathrm{T}_{\mathrm{DM}}$, while $\mathrm{AD}-12$ is a porphyritic, coarse, deformed granite, with K-feldspar, quartz, biotite and accessory muscovite with garnet of up to $1 \mathrm{~mm}$. Its $\varepsilon_{\mathrm{Nd}(630)}$ is -8.027 and its $\mathrm{T}_{\mathrm{DM}}$ is 1.68 Ga. Besides these data, Besang et al. (1977) obtained low ${ }^{87} \mathrm{Sr} /{ }^{86} \mathrm{Sr}$ ratio of 0.706 for this batholith.

\section{Perdizes Granite}

The Perdizes Granite is a NW elongated granitic body that occurs to the NW of the city of Perdizes (Figs. 1 and 3). It is intruded in garnet-mica-quartz schist and amphibolite and displays pegmatitic facies with quartz, muscovite, $\mathrm{K}$-feldspar, and tourmaline. It is porphyritic with $\mathrm{K}$-feldspar phenocrysts of up to $3 \mathrm{~cm}$, from medium to pegmatitic, white, very deformed, with quartz $(20.43 \%)$, orthoclase and microcline $(67.88 \%)$, plagioclase $(11.68 \%)$, and rare muscovite. Garnet and zircon act as accessories, and the epidote happens secondarily. This Granite is classified as syenogranite, and presents very bad metamictic zircons. There is one concordant grain of about $680 \mathrm{Ma}$, but the most reliable age of crystallisation is an average of the $6 / 8$ ages $642 \pm 59 \mathrm{Ma}$ (Fig. 21). Regarding the $\mathrm{Nd}$, it has a very high ${ }^{147} \mathrm{Sm} /{ }^{144} \mathrm{Nd}$ ratio, therefore it is not possible to calculate its $\mathrm{T}_{\mathrm{DM}}$ age. However, its $\varepsilon_{\mathrm{Nd}(642)}$ is -10.535 , indicating an origin by melting of crustal sources.

\section{Estrela do Sul Granite}

Estrela do Sul Granite is a small elliptical granitic body in Estrela do Sul (Figs. 1 and 2). It is intruded in garnetmica-quartz and amphibolite and talc-amphibole schists, in which pegmatites were seen. It is deformed at the edges

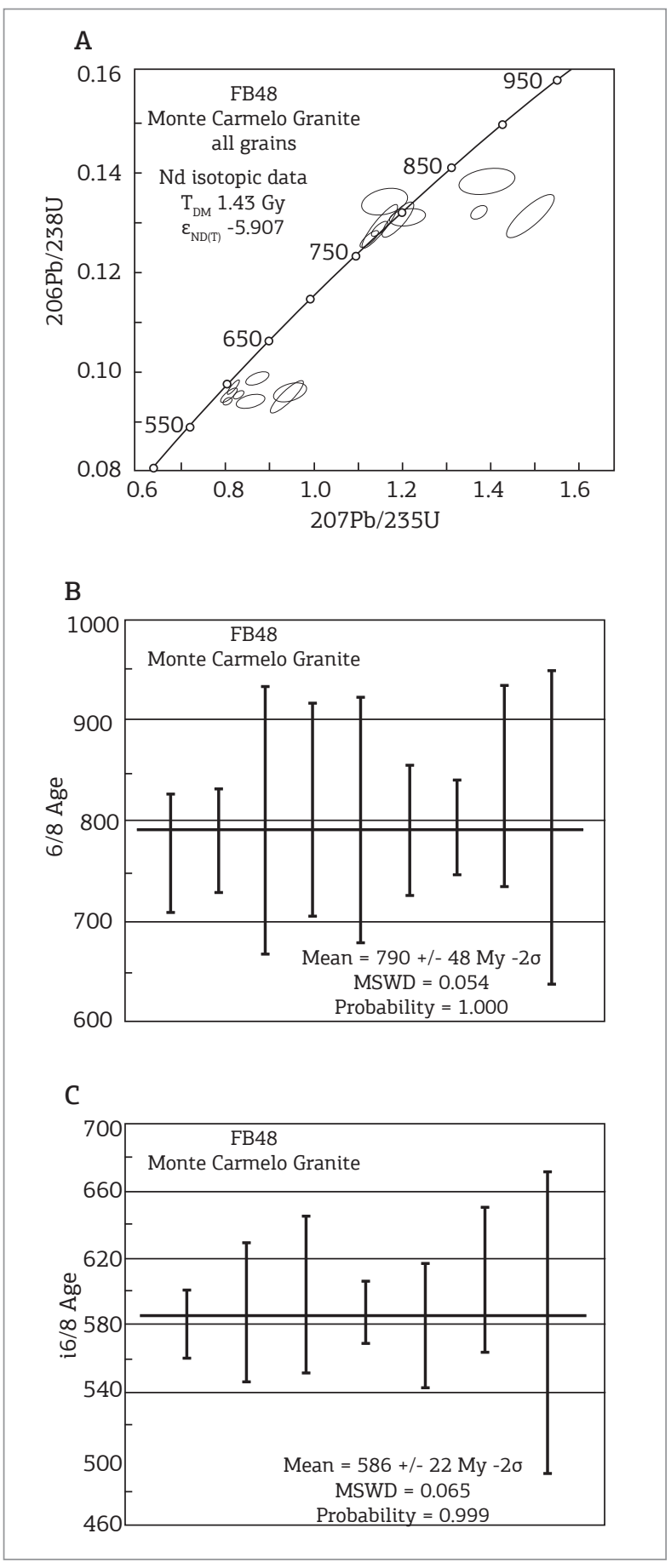

Figure 18. (A) Means of $6 / 8$ ages $\left({ }^{206} \mathrm{~Pb} /{ }^{238} \mathrm{U}\right)$ for the FB-48 sample from Monte Carmelo Granitic Complex; (B) Diagram represents crystallization age and (C) represents influence of the collisional episode.

and well preserved from deformation in the inner portions. An equigranular facies predominates, which is light grey, from fine to medium, with quartz, orthoclase and microcline, oligoclase, biotite, muscovite, and opaques and as accessories, zircon (two populations, one of prismatic and clear 
crystals and another of rounded and clear crystals), apatite, garnet and tourmaline and secondarily sericite, epidote, and carbonate. It can be classified as syenogranite, and has monzogranitic and alkali granite facies (Fig. 4). It plots in the granite field in the AnxAbxOr diagram (Fig. 7), being rich in $\mathrm{Rb}$ and $\mathrm{Cs}$ and depleted of $\mathrm{Nb}$ and $\mathrm{Y}$ (Fig. 6). Estrela do Sul Granite displays $\mathrm{Na}_{2} \mathrm{O} / \mathrm{K}_{2} \mathrm{O}$ ratio of 0.487 and normative corundum, having a peraluminous character (Fig. 8). Its strong fractionation between the LREE and HREE is represented by the $\mathrm{La}_{\mathrm{N}} / \mathrm{Yb}_{\mathrm{N}}$ ratio $=221.55$ (Fig. 9). Its $\mathrm{Eu} /$ $\mathrm{Eu}^{*}$ ratio $=0.59$ shows a weak Eu anomaly.

In a multi-element diagram (Fig. 10), it has a subduction zone component and negative anomalies of Sr, P, and Ti like the Monte Carmelo Granitic Complex samples. It plots in the field of the collisional granites in the R1xR2 (Fig. 11) and RbxY+Nb diagrams (Fig. 14). In $\mathrm{NbxY}$ (Fig. 12), it plots in the fields of the collisional and volcanic arc granites and in the HfxRb/30x3Ta diagram (Fig. 15), on the boundary among the fields of collisional and post-collisional granites.

According to Barbarin's classification, in 1999, this can be named peraluminous muscovite granite (MPG). It is on the boundaries among the I, II, and III granites (Fig. 16) in the $\mathrm{Rb} / \mathrm{ZrxSiO}_{2}$ diagram, and in the field of type II granites (Fig. 17) in the TaxNb. It presents many zircon grains $(11 / 24)$ with high levels of common lead $\left({ }^{206} \mathrm{~Pb} /{ }^{204} \mathrm{~Pb}\right)$ and another seven ones with $\mathrm{Pb}$ loss. Only six grains were used to determine a concordia age of $632.4 \pm 3.4 \mathrm{Ma}$ (Fig. 22), interpreted as the time of crystallization of this body. Its $\varepsilon_{\mathrm{Nd}(632)}$ is -7.224 and its $\mathrm{T}_{\mathrm{DM}}$ is $1.68 \mathrm{Ga}$, indicating an origin by the reworking of Mesoproterozoic crustal sources. Muscovite of this granite dated using the K-Ar method (Hasui \& Almeida 1970) provided the age of $615 \pm 1 \mathrm{Ma}$, which is seen as the cooling age of mica and is consistent with the age of crystallization and cooling of the body.

\section{Cascalho Rico Granite}

Cascalho Rico Granite is near the Cascalho Rico town (Figs. 1 and 2), forming an irregular body of small dimensions associated with Goiandira gneisses (Klein 2008). It is equigranular, medium, grey, with quartz, orthoclase, plagioclase, and biotite. Zircon, apatite, and ilmenite act as accessories, and albite and muscovite are secondary. It is a syenogranite in the QAP diagram, where $\mathrm{Q}$ is quartz; $\mathrm{A}$ is alkaline feldspar; and $\mathrm{P}$ is plagioclase (Fig. 4). It has $\mathrm{Na}_{2} \mathrm{O}$ / $\mathrm{K}_{2} \mathrm{O}$ ratio of 0.663 and high values of $\mathrm{MgO}, \mathrm{Sr}$, Th, $\mathrm{V}, \mathrm{Zr}$ and $\mathrm{Ba}$ (Figs. 5 and 6). In the AnxAbxOr diagram, it plots in the field of the granites (Fig. 7), has normative corundum, and it is peraluminous (Fig. 8).

Cascalho Rico Granite shows strong fractionation between the LREE and HREE, reaching $\mathrm{La}_{\mathrm{N}} / \mathrm{Yb}_{\mathrm{N}}=164.14$

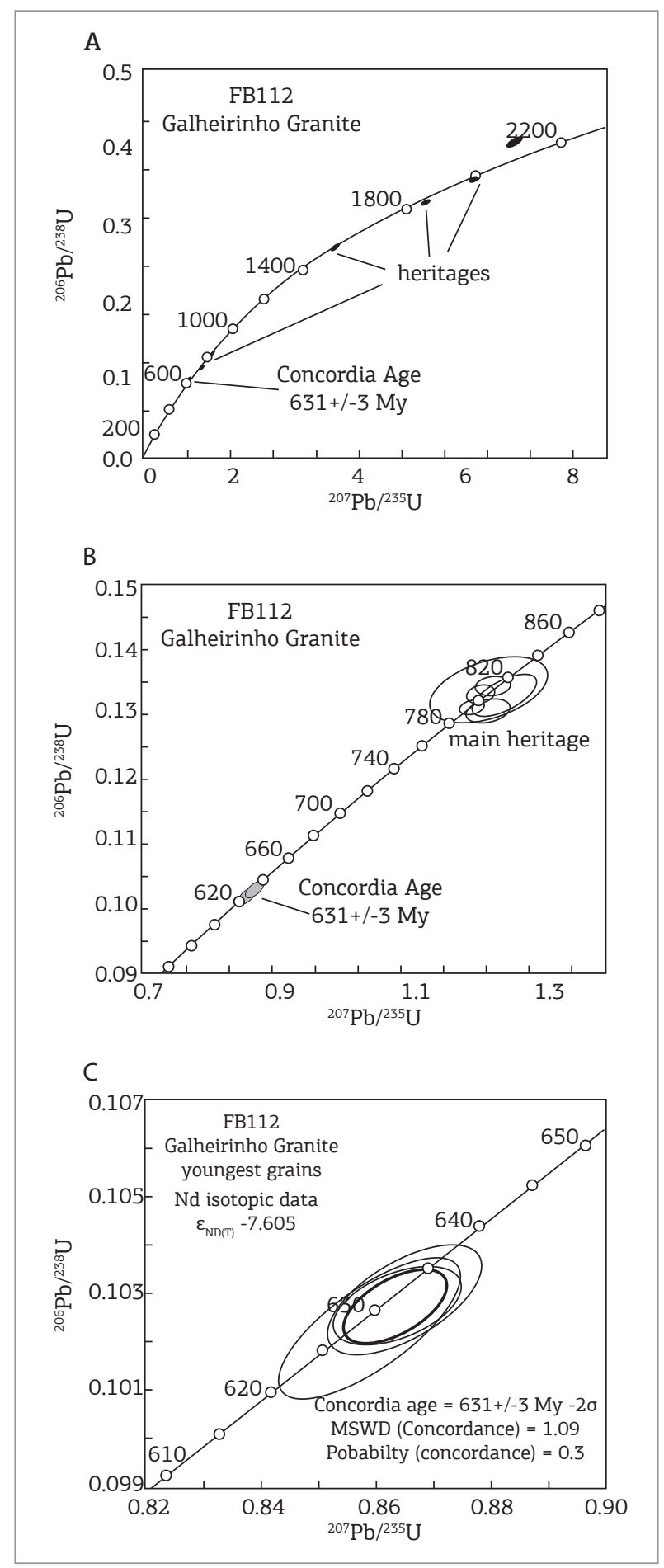

Figure 19. Tera-Wasserburg's diagram for FB-86 sample of Monte Carmelo Granitic Complex and mean of 6/8 ages.

and $\mathrm{Eu} / \mathrm{Eu} *$ ratio $=0.66$, with weak negative $\mathrm{Eu}$ anomaly (Fig. 9). In the multi-element diagram, it has subduction zone component and negative anomalies of $\mathrm{Sr}$, $\mathrm{Ti}$ and P, which are similar to the Estrela do Sul Granite (Fig. 10). This plots in the field of collisional granites in the R1xR2 
(Fig. 11) diagram, collisional granites and volcanic arc in the NbxY one (Fig. 12), and in the volcanic arc granites in the $\mathrm{RbxY}+\mathrm{Nb}$ (Fig. 14) and HfxRb/30x3Ta diagrams (Fig. 15). In the $\mathrm{Rb} / \mathrm{ZrxSiO}_{2}$ and $\mathrm{TaxNb}$ diagrams, it plots in the field of types I and III granites (Figs. 16 and 17). Most zircon grains from the FB-108 sample have very low 6/4 ratios, and other very discordant ones. The most reliable age is obtained from the average of $6 / 8$ in three grains with $633 \pm 58 \mathrm{Ma}$, given its probable age of crystallization (Fig. 23). It displays $\mathrm{T}_{\mathrm{DM}}$ of $1.48 \mathrm{Ga}$ and $\varepsilon_{\mathrm{Nd}(633)}$ of -5.909 , interpreted as a body originated by the melting of Mesoproterozoic crustal sources.

\section{Galheirinho Granite}

Galheirinho Granite outcrops badly on the BR-452 highway, between the towns of Araxá and Perdizes (Figs. 1 and 3), forming an irregular body with small dimensions, intruded in garnet-mica-quartz schist and amphibolite. This is equigranular, fine to medium, light grey, deformed, with quartz, orthoclase and microcline, plagioclase, biotite,

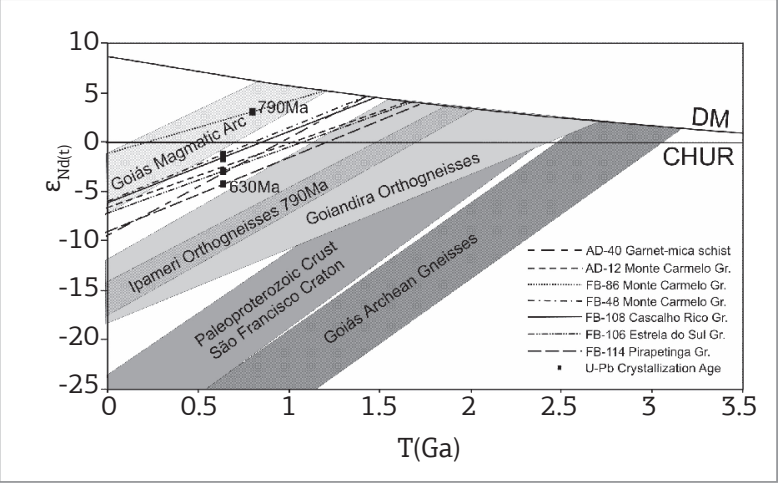

Figure 20. Epsilon $\mathrm{Nd}_{(\mathrm{t})} \mathrm{xT}$ evolution diagram for granitoids and a garnet-mica schist from Araxá Group (Goiás Magmatic Arc after Pimentel \& Fuck 1992), Goiás Archean Gnaisses and Paleoproterozoic São Francisco Craton after Pimentel et al. 1996, Goiandira and Ipameri orthogneisses after Klein (2008).

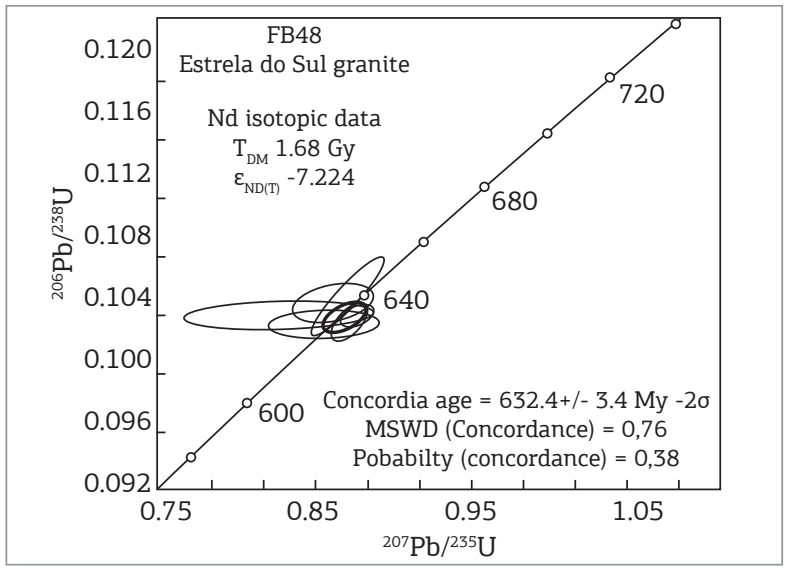

Figure 22. Concordia diagram for six zircon grains from Estrela do Sul Granite. and muscovite and zircon, apatite, garnet, and tourmaline as accessories. From the modal point of view, it is an alkali granite (Fig. 4). Its low $\mathrm{TiO}_{2}, \mathrm{CaO}, \mathrm{MgO}, \mathrm{P}_{2} \mathrm{O}_{5}, \mathrm{Sr}$ and $\mathrm{Ba}$ values are remarkable, with high $\mathrm{Rb}$ and $\mathrm{Y}$ (Figs. 5 and 6). Its $\mathrm{Na}_{2} \mathrm{O} / \mathrm{K}_{2} \mathrm{O}$ ratio is equal to 0.358 , which demonstrates the high level of $\mathrm{K}_{2} \mathrm{O}$ in relation to $\mathrm{Na}_{2} \mathrm{O}$, and the normative corundum value is the highest among the studied granites, having strong peraluminous character (Fig. 8). In the AnxAbxOr diagram, Galheirinho is classified as granite (Fig. 7). It has smooth pattern of LREE in relation to the HREE with $\mathrm{La}_{\mathrm{N}} / \mathrm{Yb}_{\mathrm{N}}$ ratio $=3.76$ and $\mathrm{Eu} / \mathrm{Eu}$ * ratio $=0.1$, indicating strong negative Eu anomaly (Fig. 9).

In the multi-element diagram, strong negative anomalies of $\mathrm{Ba}, \mathrm{Nb}, \mathrm{Sr}, \mathrm{P}$ and $\mathrm{Ti}$ are seen (Fig. 10). It plots in the field of collisional granites in the R1xR2 (Fig. 11), $\mathrm{RbxY}+\mathrm{Nb}$ (Fig. 14) and $\mathrm{Hf} \times \mathrm{R} / 30$ Tax3 diagrams (Fig. 15), collisional granites and volcanic arc in the $\mathrm{NbxY}$ diagram (Fig. 12) and in the $\mathrm{Rb} / \mathrm{ZrxSiO}_{2}$ and $\mathrm{TaxNb}$ diagrams plots in the field of type II granites (Figs. 16 and 17). According to Barbarin's classification, in 1999, it can be called MPG. Some zircon grains of the FB-112 sample

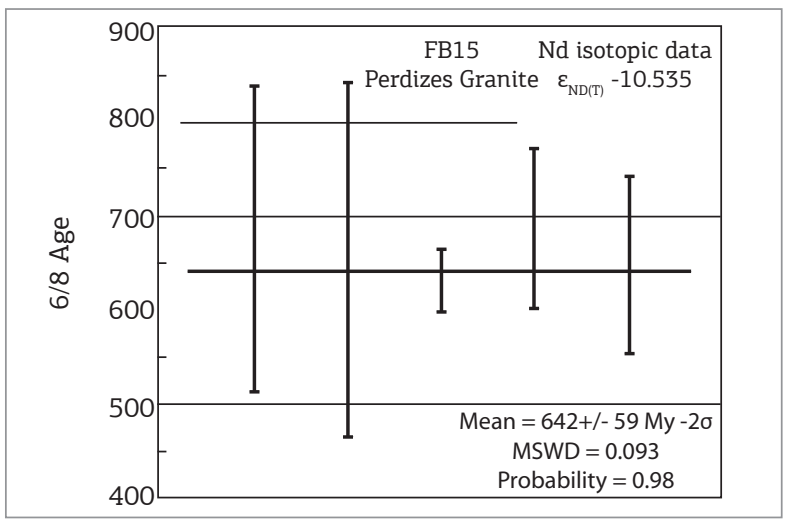

Figure 21. Mean of 6/8 ages for five zircon grains from Perdizes Granite.

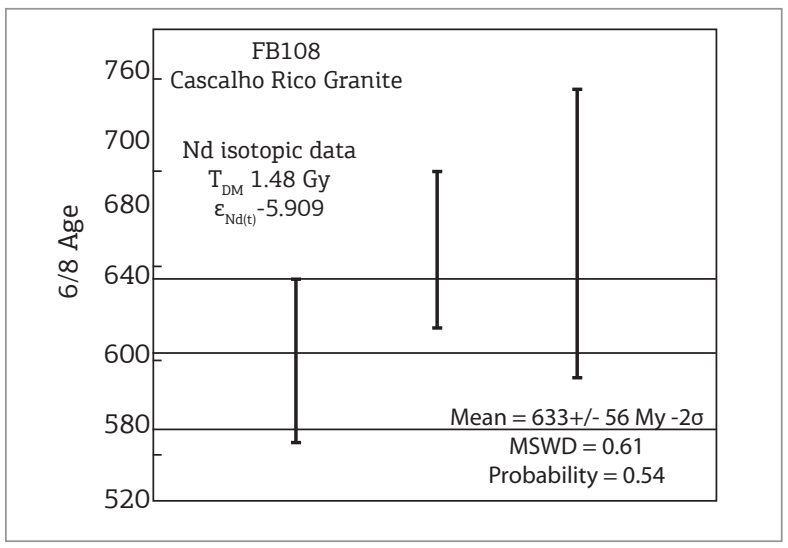

Figure 23. Mean of $6 / 8$ ages $\left({ }^{206} \mathrm{~Pb} /{ }^{238} \mathrm{U}\right)$ for three zircon grains from Cascalho Rico Granite. 
have high common $\mathrm{Pb}$. However, four ones show a good concordia age of $631 \pm 3 \mathrm{Ma}$ (Fig. 24), which indicates the crystallization time of this body. Several zircon grains show Paleoproterozoic inheritance (1836.4 and 2207.1 $\mathrm{Ma}$ ), Mesoproterozoic (1505.6 and $1552.6 \mathrm{Ma}$ ), and Neoproterozoic $(-800 \mathrm{Ma})$, being the latter the most significant one. The ${ }^{147} \mathrm{Sm} /{ }^{144} \mathrm{Nd}$ ratio is quite high not making it possible to calculate a model age. Its $\varepsilon_{\mathrm{Nd}(631)}$ is -7.605 indicating reworking of crustal sources. Among the bodies investigated, this is the one that shows the oldest heritages.

\section{Pirapetinga Granite}

Pirapetinga Granite is a folded tabular body that occurs on the NE border of the Araxá Nappe (Figs. 1 and 3). It is intrusive into garnet-mica-quartz schist and amphibolite, and presents abundant pegmatitic facies constituted of quartz, muscovite, K-feldspar, and tourmaline. It is equigranular, medium, light grey, deformed, with quartz, orthoclase and microcline, muscovite and biotite, and rare grains of plagioclase. Zircon, tourmaline and apatite are acessories. It is an alkali granite from the modal point of view (Fig. 4). Chemically, it stands out for its high level of $\mathrm{K}_{2} \mathrm{O}$, high $\mathrm{Rb}$ and very low $\mathrm{Na}_{2} \mathrm{O}$, which reflected in the low $\mathrm{Na}_{2} \mathrm{O} / \mathrm{K}_{2} \mathrm{O} 0.149$ ratio (Figs. 5 and 6) and in the AnxAbxOr diagram, where it plots near the apex of Or, being compatible with the high modal percentage of orthoclase (51.38\%), as seen in Fig. 7.

This Granite has high normative corundum and peraluminous character (Fig. 8). Its $\mathrm{La}_{\mathrm{N}} / \mathrm{Yb}_{\mathrm{N}}=23.42$ ratio reflects a low-enriched pattern in LREE compared to $\mathrm{HREE}$ and its $\mathrm{Eu} / \mathrm{Eu}{ }^{*}$ ratio $=0.49$, indicating a significant negative Eu anomaly (Fig. 9). In the multi-element diagram, its negative anomalies of $\mathrm{Nb}, \mathrm{Sr}$ and $\mathrm{Ti}$ stand out (Fig. 10). It plots on the boundary between the fields of collisional and anorogenic granites in the R1xR2 diagrams (Fig. 11), and volcanic arc and collisional granites in the YxNb (Fig. 12) and HfxR/30x3Ta diagrams (Fig. 15) and collisional in $\mathrm{RbxY}+\mathrm{Nb}$ (Fig. 14). According to Barbarin's classification, in 1999, it can be called MPG. In the Rb/ $\mathrm{ZrxSiO}_{2}$ and $\mathrm{ZrxTaxNb}$ diagrams (Figs. 16 and 17), it plots on the boundary among the fields of granites types I, II and III. Its zircons present high isotopic imbalances, which may indicate hydrothermal alteration and unreliability in the chemical analysis. The FB-114 sample has only eight zircon grains with reasonable results, also there is the incorporation of $\mathrm{Pb}$ (reverse) with a discordia age of $623 \pm 41 \mathrm{Ma}$. However, there is a concordant grain of about $630 \mathrm{Ma}$ (Fig. 25) that could be its crystallization age. Its $\mathrm{T}_{\mathrm{DM}}$ is $1.73 \mathrm{Ga}$, the highest model age among the studied bodies. Its $\varepsilon_{\mathrm{Nd}(630)}$ is -8.934 , indicating reworking of crustal sources.

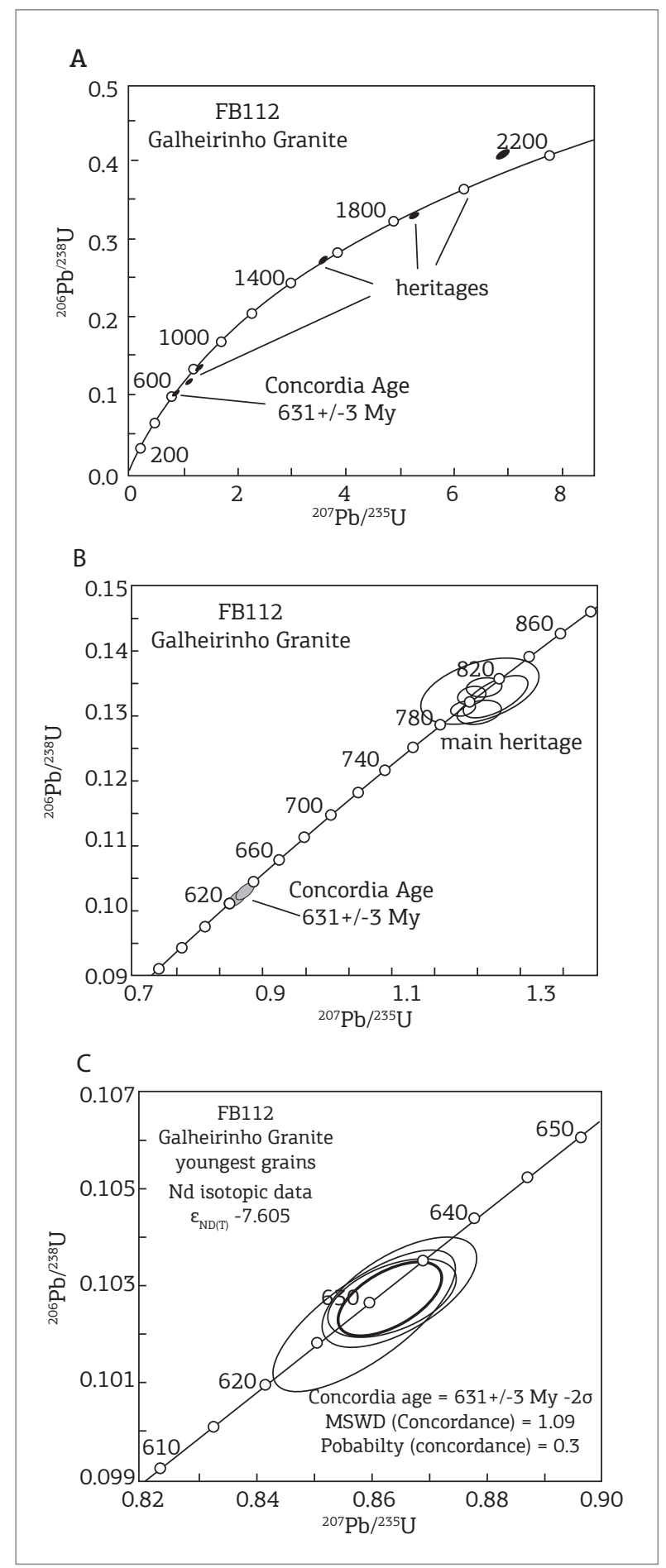

Figure 24. (A) Concordia diagrams for zircons from Galheirinho Granite; (B) More significant heritages; (C) Concordia age.

\section{Serra Velha Granite}

Serra Velha Granite is a small NW lenticular body situated to the North of Araxá (Figs. 1 and 3). It is intruded in garnet-mica-quartz schist and amphibolite, and is intensely deformed with concordant tectonic foliation with the 
hosting rocks, presenting gneiss aspect. It is equigranular, fine to medium, light grey to white, with quartz, orthoclase, microcline, plagioclase, biotite and muscovite, and garnet, tourmaline, monazite, apatite and zircon as accessories. Sericite, biotite, albite and chlorite secondary act as retrometamorphic minerals. From the modal point of view, it is a granite (Fig. 4). Samples of this body analyzed by Seer (1999) are classified as granite in the AnxAbxOr diagram (Fig. 7), presenting normative corundum and being peraluminous (Fig. 8), with low Ba, V and Sr and high Y (Fig. 6). It may also have been formed by melting of pelitic metasediments. They present low $\mathrm{La}_{\mathrm{N}} / \mathrm{Yb}_{\mathrm{N}}$ ratios ranging from 2.13 to 6.8 and $\mathrm{Eu} / \mathrm{Eu}^{*}$ ones from 0.27 to 0.54 (Fig. 9). In the multi-element diagram, its resemblance to the Galheirinho Granite is remarkable (Fig. 10). It plots in the field of collisional granites in the R1xR2 (Fig. 11) and $\mathrm{RbxY}+\mathrm{Nb}$ diagrams (Fig. 14), collisional granites and volcanic arc in the NbxY diagram (Fig. 12). According to Barbarin's classification, in 1999, it can be called MPG. Zircon dating by isotopic dilution - thermal ionization mass spectrometer ID-TIMS (Valeriano et al. 2004a) provided concordant age of $637 \pm 1 \mathrm{Ma}$, considered the crystallization age of this body (Fig. 26). Some zircon inherited from the source area provided the age of $830 \mathrm{Ma}$.

\section{Tamanduá Granite}

Tamanduá Granite (FB-116 sample) is an NW elongated body in the Araxá Nappe, beside the Serra Velha Granite (Figs. 1 and 3). It is intrusive into garnet-mica-quartz schist, quartzite and amphibolite and has pegmatitic facies formed by quartz, muscovite, K-feldspar, and tourmaline. It has abundant xenoliths of amphibolite, schist, and quartzite on its edge. It is dominantly equigranular, medium, light grey, deformed, with quartz $(26.76 \%)$, orthoclase and microcline (45.49\%), plagioclase $(17.15 \%)$, biotite $(6.17 \%)$, muscovite $(4.41 \%)$. Apatite and zircon are accessories. Secondary muscovite occurs on the K-feldspar. In the QAP diagram, it is classified as syenogranite. Isotopically, its zircons are completely unbalanced, not being possible to obtain the U-Pb age. It is believed that this body has undergone strong post-magmatic hydrothermal alteration, a fact corroborated by the abundant presence of pegmatites. Taking into account only the $\mathrm{Nd}$ data and using a crystallization age of $637 \mathrm{Ma}$ ( $\sim$ Serra Velha granite), its $\varepsilon_{\mathrm{Nd}(637)}$ is -5.728 with $\mathrm{T}_{\mathrm{DM}}$ of $1.56 \mathrm{Ga}$, values that match the other samples.

\section{DISCUSSION}

Field studies and petrographic, geochemical, and geochronological data make it possible to claim that the intrusive granites into the metasedimentary, mafic and ultramafic metaigneous rocks of the Araxá Group, between Araxá and Cascalho Rico towns, represent three episodes of Neoproterozoic granitic magmatism, each one related to a distinct environment. The first one is related to the Quebra Anzol Granite, aged 833 Ma, which was originated in within-plate environment and is associated with a large volume of amphibolite. The mafic rocks and granite can be interpreted as an accreted terrain that would represent the first magmatic manifestations in a within-plate environment. Tholeiitic amphibolites associated with the Araxá Group in the Anápolis region (Piuzana et al. 2003) aged $838 \pm 20 \mathrm{Ma}$ and $\varepsilon_{\mathrm{Nd}}+5.1$ represent a fragment of oceanic crust of the SBB, and may be co-related to the Araxá Nappe and to the region of Ipameri-Catalão, which are characterized as tholeiites of mid-ocean ridge (Seer 1999, Klein, 2008). This age represents therefore an extensional event in SBB and is compatible with that of the Quebra Anzol Granite.

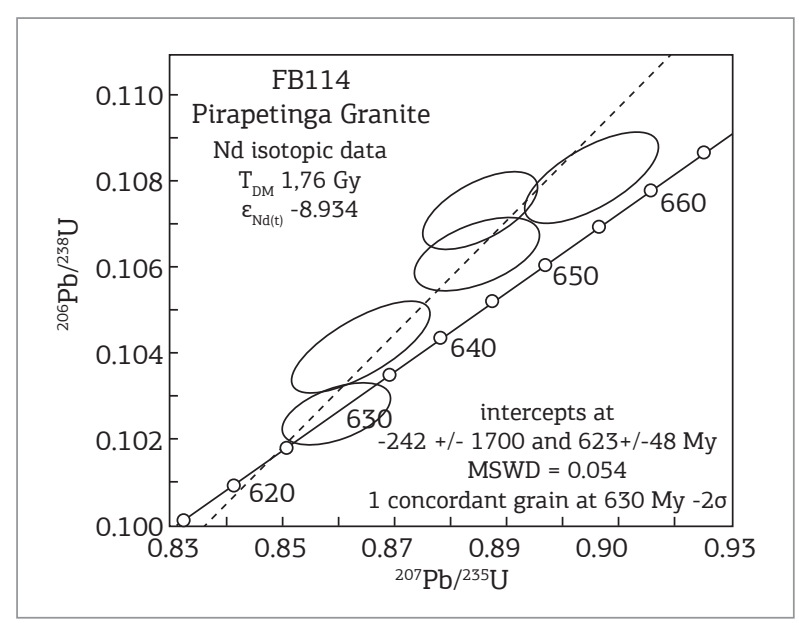

Figure 25. Concordia age for one zircon grain from Pirapetinga Granite.

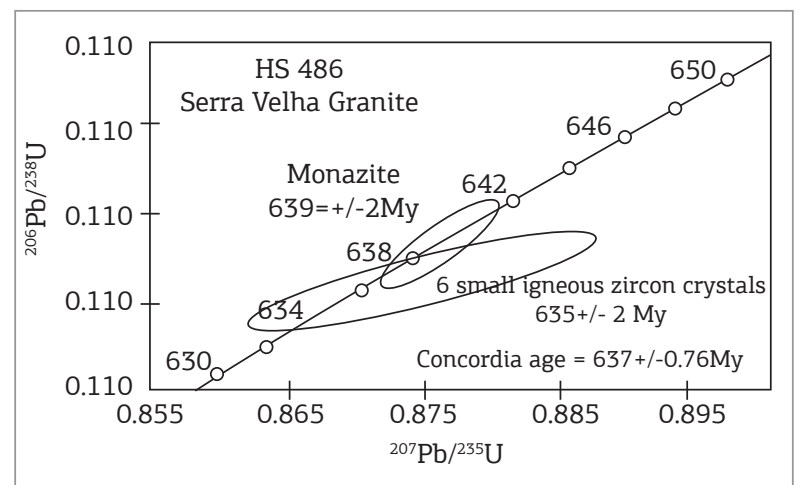

Figure 26. Concordia diagram for monazite and zircon grains from Serra Velha Granite (Valeriano et al. 2004a). 
Of a bit younger age, around $790 \mathrm{Ma}$, are the Pires Belo and Davinópolis granites in Catalão, which Klein (2008) interpreted as being of extensional origin. Such fact suggests that the events generating granites of this age would have persisted for at least $48 \mathrm{Ma}$. In contrast, a little to the NW, Pimentel et al. (1992) obtained the age of $790 \mathrm{Ma}$ for the Maratá Granite interpreting it as being collisional. The second magmatic episode is represented by granites aged 790 Ma. Geological, geochemical, and isotopic evidence suggest that the Monte Carmelo Granitic Complex and associated amphibolites may represent the root of a magmatic arc, whose volcanosedimentary counterpart would be represented by the volcanosedimentary sequence of Abadia dos Dourados. The $\mathrm{Nd}$ isotopic data $\left(\varepsilon_{\mathrm{Nd}(790)}\right.$ of -2.218 and $\mathrm{T}_{\mathrm{DM}}$ of 1.29) reinforce this hypothesis, showing its similarity to the isotopic pattern of the rocks of Goiás Magmatic Arc (Fig. 20, Laux et al. 2005). Associated with this complex, bodies of peraluminous granites happen with garnet and muscovite, typically collisional and may represent the melting of arc granites around $630 \mathrm{Ma}$, since they contain zircon populations inherited from $790 \mathrm{Ma}$ and similar patterns of REE. The third episode between 642 and $630 \mathrm{Ma}$ is represented by the collisional magmatism, which is already well characterized in the region and with metamorphic peak between 640 and $637 \mathrm{Ma}$ (Seer et al. 2010). This is represented by granitic bodies of Serra Velha, Tamanduá, Pirapetinga, Galheirinho, Perdizes, Estrela do Sul and Cascalho Rico, generated from the reworking of continental neo and mesoprotrozoic crusts. These ages are similar to the Goiandira orthogneisses (Klein 2008) to the Firminópolis and Palminópolis ones, and in this case also to the evolution pattern of $\mathrm{Nd}$ (Laux et al. 2005) and to lenticular anatetic bodies of the Passos Nappe (Valeriano et al. 2004a).

The $\mathrm{Nd}$ evolution pattern of the collisional granites of Araxá and Monte Carmelo is very similar to the metassediments of the Araxá Group, both for the sample of Volcanosedimentary Sequence Abadia dos Dourados and for that of Anápolis and Goiânia region (Pimentel et al. 1999b). However, very different from the patterns of the Goiandira - Ipameri gneisses, indicating that the Araxá and Monte Carmelo granites may have been originated by the melting of sediments with low crustal residence, perhaps having been generated in forearc basins. An exotic terrain of magmatic arc formed at the end of the Mesoproterozoic in SBB, with an age of $1.2 \mathrm{Ga}$, represented by the Nova Aurora orthogneisses, was recently characterized (Klein 2008). This age shows the existence of a fourth and the oldest episode of granitic magmatism in the SBB. In the tectonic evolution context of SBB, the data presented here conform to the original idea of Pimentel et al. (1999a) and
Laux et al. (2005), who discussed the possibility that several intra-oceanic arcs would have developed independently and were accreted during the evolution of SBB. But, it also reaffirms the possibility of tectono-stratigraphic terrain accretion that differ in both their ages and geochemical and isotopic patterns.

The collisional process occurred between 640 and $630 \mathrm{Ma}$, well marked by granitic magmatism, joined several terrains of within-plate, ophiolitic melanges and of magmatic arc and generated sincolisional sedimentary (Dias et al. 2011) or forearc basins (Pimentel et al. 2011), later deformed, juxtaposing themselves in nappe systems through extensive subhorizontal and subvertical shear zones, whose exhumation and cooling would have occurred up to $605 \mathrm{Ma}$ (Valeriano et al. 2004b).

\section{CONCLUSIONS}

The data presented show the existence of three episodes of granites generation in distinct tectonic environments in the SBB, between Araxá and Cascalho Rico, Minas Gerais State, in Brazil. The earliest episode is represented by the Quebra Anzol Granite, aged $833 \mathrm{Ma}$, with low ${ }^{87} \mathrm{Sr} /{ }^{86} \mathrm{Sr}$ ratio of 0.706 and $\varepsilon_{\mathrm{Nd}(833)}=-3.98$. Its peralcalinity, high $\mathrm{SiO}_{2}, \mathrm{Na}_{2} \mathrm{O}, \mathrm{MnO}, \mathrm{Nb}, \mathrm{Y}$ and $\mathrm{Co}$ values and low $\mathrm{Sr}$ and $\mathrm{Ba}$, absence of anorthite and presence of acmite and diopside indicate that it may have been originated from mantle sources with little crustal contamination, although it was affected by post-magmatic hydrothermal alteration and mylonitization. In the tectonic discrimination diagrams, it associates with the field of within-plate granites. The second magmatic episode is represented by Monte Carmelo Granitic Complex, aged $790 \mathrm{Ma}$, and partly remobilized around $630 \mathrm{Ma}$. It is metaluminous to peraluminous, with low ${ }^{87} \mathrm{Sr} /{ }^{86} \mathrm{Sr}$ ratios of 0.706 , high fractionation of LREE in relation to HREE and negative anomalies of $\mathrm{Nb}, \mathrm{Ta}$, Sr, $\mathrm{P}$ and Ti. Its $\varepsilon_{\mathrm{Nd}(790)}$ is -2.218 and its $\mathrm{T}_{\mathrm{DM}}$ is $1.29 \mathrm{Ga}$, indicating an origin from juvenile sources at the end of the Mesoproterozoic. Its $\mathrm{Nd}$ evolution curve in relation to time is similar to that of Goiás Magmatic Arc granites.

From the tectonic point of view, the granites aged $790 \mathrm{Ma}$ were formed in a pre-collisional magmatic arc environment, and they are associated with collisional granites generated around $630 \mathrm{Ma}$. These are products of remobilization of older granites, a fact which is proved both by the presence of inherited zircons of $790 \mathrm{Ma}$ and similarity of the REE patterns, although other older crustal components may also have participated in the process as demonstrated by Mesoproterozoic zircons and Nd model ages. The last episode is represented by Serra Velha, Tamanduá, Pirapetinga, 
Galheirinho, Perdizes, Estrela do Sul, and Cascalho Rico granites whose crystallization ages range from 642 to $630 \mathrm{Ma}$. They are peraluminous granites, mostly with muscovite and garnet, as well as tourmaline, with negative $\varepsilon_{\mathrm{Ndr}}$, model ages of $\mathrm{Nd}$ from Neo to Mesoproterozoic, enrichment patterns of LREE in relation to HREE variable according to the melting of igneous or sedimentary sources. In this case, with weak enrichment in REE and strong negative anomalies of Eu. In discriminant diagram, they plot in the field of collisional granites. These three episodes of granitic magmatism of different ages show that the collisional process that occurred around 640 to $630 \mathrm{Ma}$ in this area of the SBB amalgamated tectono -stratigraphic terrains generated in within-plate, forearc, and magmatic arc tectonic environments.

\section{ACKNOWLEDGEMENTS}

This study was financially supported by Fundação de Amparo à Pesquisa do Estado de Minas Gerais - FAPEMIG (process 139-04) to which the authors are grateful. We thank professors José Affonso Brod (Federal University of Goiás) for his support in carrying out some chemical analysis of granites, Reinhard Adolf Fuck (University of Brasília) for his support in performing some Sm-Nd analysis, Sergio Luis Junges (Federal University of Rio Grande do Sul) for the discussions on the geochronological data, and Valda Sánchez (Araxá Cultura Inglesa) for the English version of the original manuscript. We are also grateful to the BJG proofreaders.

\section{REFERENCES}

Barbosa O. 1937. Geologia do Município de Araxá, Minas Gerais. Mineração e Metalurgia, p. 247-248.

Barbarin B. 1999. A review of the relationships between granitoid types, their origins and their geodynamic environments. Lithos, 46:605-626

Barbosa O., Braun O.P.G., Dyer R.C., Cunha C.A.B.R. 1970. Geologia da região do Triângulo Mineiro. Rio de Janeiro: Departamento Nacional da Produção Mineral/Divisão de Fomento da Produção Mineral, Boletim 136, $140 \mathrm{p}$.

Batchelor R.A. \& Bowden P. 1985. Petrogenetic interpretation of granitoid rock series using multicationic parameters. Chemical Geology, 48:43-55.

Besang C., Eberle W., Lahner L., Lenz H., Mollat H., Müller P., Paulsen S. 1977. Datação radiométrica em rochas de MG e ES. Belo Horizonte, Serviço Geológico do Brasil - CPRM, 41 p.

Brod J.A., Leonardo S.O.H., Meneses P.R., Albuquerque M.A.C., Almeida R., Blanco S.B., Cardoso F.B.F, Romão P.A., Tallarico F.H.B., Thomsen F.P.R. 1991. Geoquímica da Sequência Vulcano Sedimentar de Abadia dos Dourados e Complexo Chapada das Perdizes, Triângulo Mineiro - MG. Revista Escola de Minas, 45(1-2):164-166.

Bühn B., Pimentel M.M., Matteini M., Dantas E.L. 2009. High spatial resolution analysis of $\mathrm{Pb}$ and $\mathrm{U}$ isotopes for geochronology by laser ablation multi-collector inductively coupled plasma mass spectrometry (LA-MC-ICP-MS). Anais da Academia Brasileira de Ciências, 81:1-16.

Della Giustina M.E.S., Pimentel M.M., Ferreira Filho C.F., Maia de Holanda M.H.B. 2011. Dating coeval mafic magmatism and ultrahigh temperature metamorphism in the Anápolis-Itauçu Complex, Central Brazil. Lithos, 124:82-102.

DePaolo D.J. 1981. A neodymium and strontium isotopic study of the Mesozoic calcalkaline granitic batholiths of the Sierra Nevada and Peninsular Ranges, California. Journal of Geophysical Research, 86:10470-10488.

Dias P.H.A., Noce C.M., Pedrosa-Soares A.C., Seer H.J., Dussin I.A., Valeriano C.M., Kuchenbecker M. 2011. O Grupo Ibiá (Faixa Brasília Meridional): evidências isotópicas Sm-Nd e U-Pb de bacia colisional tipo flysch. Geonomos, 19(2):90-99.
Gioia S.M., Pimentel M.M. 2000. The Sm-Nd isotopic method in the geochronology laboratory of the University of Brasília. Anais da Academia Brasileira de Ciências, 72(2):219-254

Harris N.B.W., Pearce J.A., Tindle A.G. 1986. Geochemical characteristics of collision-zone magmatism. In: Coward M.P. \& Ries A.C. (eds) 1986. Collision tectonics. Blackwell Scientific Publications, Oxford. Geological Society Special Publication, 19:67-81.

Hasui Y. \& Almeida F.F.M. 1970. Geocronologia do Centro-Oeste Brasileiro. Boletim da Sociedade Brasileira de Geologia, 19(1):7-26.

Laux J.H., Pimentel M.M., Dantas E.L., Armstrong R., Junges S.L. 2005. Two Neoproterozoic crustal accretion events in the Brasília Belt, Central Brazil. Journal of South American Earth Sciences, 18:183-198.

Klein P.B.W. 2008. Geoquímica de Rocha Total, Geocronologia de U-Pb e Geologia Isotópica de Sm-Nd das Rochas Ortognáissicas e Unidades Litológicas Associadas da Região Ipameri - Catalão (Goiás). Tese de Doutorado, Instituto de Geociências, Universidade de Brasília, 155 p.

Pearce J.A., Harris N.B.W., Tindle A.G. 1984. Trace element discrimination diagrams for the tectonic interpretation of granitic rocks. Journal of Petrology, 25:956-983.

Pereira A.D.C, Souza Jr J.J., Fonseca E.G., Oliveira F.C., Ortiz L.R.C., Freire F.A., Carmo U.F., Braz E.R.C., Marques N.M.G., Reis V.A., Siga Jr. O. 1983. Geologia da Folha SE.23 - Belo Horizonte. Levantamento de Recursos Naturais. Rio de Janeiro, DNPM/RADAMBRASIL.

Pimentel M.M. \& Fuck R.A. 1992. Neoproterozoic crustal accretion in central Brasil. Geology, 20:375-379.

Pimentel M.M., Heaman L., Fuck R.A. 1992. Idade do meta-riolito da sequiência Maratá, Grupo Araxá, Goiás: estudo geocronológico pelos métodos U-Pb em zircão, Sm-Nd e Rb-Sr. Anais da Academia Brasileira de Ciências, 64:19-28.

Pimentel M.M., Fuck R.A., Botelho N.F. 1999a. Granites and the geodynamic history of the Neoproterozoic Brasília Belt, Central Brazil: a review. Lithos, 46(3):1-21

Pimentel M.M., Fuck R.A., Fischel D.P. 1999b. Estudo isotópico Sm-Nd regional da Porção Central da Faixa Brasília, Goiás: implicações para idade e origem dos granulitos do Complexo Anápolis-Itauçú e rochas metassedimentares do Grupo Araxá. Revista Brasileira de Geociências, 29(2):271-276 
Pimentel M.M., Rodrigues J.B., DellaGiustina M.E.S., Junges S., Matteini M., Armstrong R. 2011. The tectonic evolution of the Neoproterozoic Brasília Belt, central Brazil, based on SHRIMP and LA-ICPMS U-Pb sedimentary provenance data: A review. Journal of South American Earth Science, 31:345-357.

Piuzana D., Pimentel M.M., Fuck R.A., Armstrong R. 2003. SHRIMP $\mathrm{U}-\mathrm{Pb}$ and $\mathrm{Sm}-\mathrm{Nd}$ data for the Araxá Group and associated magmatic rocks: constraints for the age of sedimentation and geodynamic context of the southern Brasília Belt, central Brazil. Precambrian Research, 125:139-160.

Rollinson H.R. 1993. Using Geochemical Data: evaluation, presentation, interpretation. New York, Longman, Scientific \& Technical, $352 \mathrm{p}$.

Seer H.J. 1999. Evolução Tectônica dos Grupos Araxá, Ibiá e Canastra na sinforma de Araxá, Araxá, Minas Gerais. Tese de Doutorado, Instituto de Geociências, Universidade de Brasília, 267 p.

Seer H.J. \& Dardenne M.A. 2000. Tectonostratigraphic terrane analysis on Neoproterozoic times: the case study of the Araxá Synform, Minas Gerais, Brazil - implications to the final collage of the Gondwanaland. Revista Brasileira de Geociências, 30(1):78-81.

Seer H.J., Brod J.A., Fuck R.A., Pimentel M.M., Boaventura G.R., Dardenne M.A. 2001. Grupo Araxá em sua área tipo: um fragmento de crosta oceânica Neoproterozóica na Faixa de Dobramentos Brasília. Revista Brasileira de Geociências, 31(3):385-396.

Seer H.J., Brod J.A., Valeriano C.M., Fuck R.A. 2005. Leucogranitos intrusivos no Grupo Araxá: registro de um evento magmático durante colisão Neoproterozóica na porção meridional da Faixa Brasília. Revista Brasileira de Geociências, 35(1):33-42.
Seer H.J., Moraes L.C., Carneiro A.C. 2007. Geologia e deformação do Grupo Araxá na região de Estrela do Sul-Monte Carmelo-Abadia dos Dourados, Minas Gerais. In: SBG, Simpósio de Geologia, Sudeste, 10:37.

Seer H.J., Moraes L.C., Dantas E.L., Binatti I. 2008. Geologia e geocronologia U-Pb do Granito Estrela do Sul, Faixa Brasília, Minas Gerais. In: SBG, Congresso Brasileiro de Geologia, 44, CD-Rom.

Seer H.J., Moraes L.C., Junges S.L. 2010. Novos dados U-Pb e SmNd de rochas granitóides do Grupo Araxá, Faixa Brasília Meridional, entre a região de Araxá e Monte Carmelo, MG. In: SBG, Congresso Brasileiro de Geologia, 45, CD-Rom.

Simões L.S.A. \& Navarro G. 1996. Estruturação da Faixa Brasília na região de Araxá, MG. In: SBG, Congresso Brasileiro de Geologia, 39:92-95.

Simões L.S.A. 2005. Compartimentos Crustais do Domínio Interno da Faixa Brasília no Sul de Goiás. Tese de Livre-Docência, Departamento de Petrologia e Metalogenia/Universidade Estadual Paulista "Júlio de Mesquita Filho", Rio Claro, 110 p.

Valeriano C.M., Machado N., Simonetti A., Valladares C.S., Seer H.J., Simões L.S.A. 2004a. U-PB Geochronology of the southern Brasília Belt (SE Brazil): sedimentary provenance, Neoproterozoic orogeny and assembly of West-Gondwana. Precambrian Research, 130(1-4):27-55.

Valeriano C.M., Dardenne M.A., Fonseca M.A., Simões L.S.A., Seer H.J. 2004b. A evolução tectônica da Faixa Brasília. In: Mantesso-Neto V., Bartorelli A., Carneiro C.D.R., Brito-Neves B.B (eds) Geologia do Continente Sul-Americano: Evolução da Obra de Fernando Flávio Marques de Almeida. São Paulo, Beca, p. 575-592.

Arquivo digital disponível on-line no site www.sbgeo.org.br 